\title{
Heterodimetallic Germanium(IV) Complex Structures with Transition Metals
}

\author{
Fa-Nian Shi, ${ }^{\dagger}$ Luís Cunha-Silva, ${ }^{\dagger}$ Michaele J. Hardie, ${ }^{\dagger}$ Tito Trindade,${ }^{\dagger}$ Filipe A. Almeida Paz, ${ }^{\dagger}$ and \\ João Rocha ${ }^{*}$,
}

Department of Chemistry, University of Aveiro, CICECO, 3810-193 Aveiro, Portugal and School of Chemistry, University of Leeds, Leeds LS2 9JT, United Kingdom

Received March 15, 2007

\begin{abstract}
The hydrothermal synthesis and structural characterization of a number of complex compounds containing the divalent tris(oxalato- $\left.O, O^{\prime}\right)$ germanate anion, $\left[\mathrm{Ge}\left(\mathrm{C}_{2} \mathrm{O}_{4}\right)_{3}\right]^{2-}$, or the neutral bis(oxalate- $\left.O, O^{\prime}\right)$ germanium fragment, $\left[\mathrm{Ge}\left(\mathrm{C}_{2} \mathrm{O}_{4}\right)_{2}\right]$, with transition-metal $(\mathrm{M})$ cationic complexes of $1,10^{\prime}$-phenanthroline (phen) is reported: [M(phen $\left.)_{3}\right]^{-}$ $\left[\mathrm{Ge}\left(\mathrm{C}_{2} \mathrm{O}_{4}\right)_{3}\right] \cdot \mathrm{HH}_{2} \mathrm{O}$ [where $\mathrm{M}^{2+}=\mathrm{Cu}^{2+}(\mathbf{1} \mathbf{a}$ and $\mathbf{1 b}), \mathrm{Fe}^{2+}(\mathbf{2 a}$ and $\mathbf{2 b}), \mathrm{Ni}^{2+}(\mathbf{3}), \mathrm{Co}^{2+}(\mathbf{4}) ; x=0.2$ for $\mathbf{2 b}$ ], [MGe(phen) $)_{2}\left(\mu_{2}-\mathrm{OH}\right)_{2}\left(\mathrm{C}_{2} \mathrm{O}_{4}\right)_{2}$ ] [where $\mathrm{M}^{2+}=\mathrm{Cd}^{2+}(5)$ and $\left.\mathrm{Cu}^{2+}(6)\right]$. The isolation of two polymorphs with $\mathrm{Cu}^{2+}(1 \mathrm{a}$ and $1 \mathrm{~b})$ and other pseudo-polymorphs for $\mathrm{Fe}^{2+}(\mathbf{2 a}$ and $\mathbf{2 b}$ ) was rationalized based on slightly different molar ratios for the starting materials. All compounds have been characterized using EDS, SEM, vibrational spectroscopy (FT-IR and FT-Raman), thermogravimetry, and $\mathrm{CHN}$ elemental composition and their structure determined on the basis of single-crystal X-ray diffraction studies. The crystal packing of the different chemical moieties for each series of compounds was discussed on the basis of the various intermolecular interactions present (strong $\mathrm{C}-\mathrm{H} \cdots \pi$ and weak $\mathrm{C}-\mathrm{H} \cdots \mathrm{O}$ hydrogen-bonding interactions, $\mathrm{C}-\mathrm{H} \cdots \pi$ and $\pi-\pi$ contacts).
\end{abstract}

\section{Introduction}

In recent years, research on crystalline organic-inorganic hybrid oxalates has gained great interest in materials science due to the structural diversity of these compounds which range from discrete complexes ${ }^{1-4}$ to $1 \mathrm{D}$ chains, ${ }^{5-7} 2 \mathrm{D}$ layers, ${ }^{8,9}$ and 3D open frameworks. ${ }^{10-12}$ Moreover, some of

* To whom correspondence should be addressed. Phone: $(+351) 234$ 370730. Fax: (+351) 234 370084. E-mail: rocha@dq.ua.pt.

University of Aveiro.

$\doteqdot$ University of Leeds.

(1) Cangussu, D.; Stumpf, H. O.; Adams, H.; Thomas, J. A.; Lloret, F.; Julve, M. Inorg. Chim. Acta 2005, 358, 2292-2302.

(2) Armentano, D.; De Munno, G.; Lloret, F.; Julve, M. CrystEngComm 2005, 7, 57-66.

(3) Carranza, J.; Grove, H.; Sletten, J.; Lloret, F.; Julve, M.; Kruger, P. E.; Eller, C.; Rillema, D. P. Eur. J. Inorg. Chem. 2004, 4836-4848.

(4) Vaidhyanathan, R.; Natarajan, S.; Rao, C. N. R. Dalton Trans. 2001, 699-706.

(5) Zhang, Q. Z.; Yang, W. B.; Lu, C. Z. J. Chem. Crystallogr. 2006, 36, $225-228$.

(6) Yang, S. H.; Li, G. B.; Tian, S. J.; Liao, F. H.; Lin, J. H. Eur. J. Inorg. Chem. 2006, 2850-2854.

(7) Garcia-Couceiro, U.; Castillo, O.; Luque, A.; Garcia-Teran, J. P.; Beobide, G.; Roman, P. Eur. J. Inorg. Chem. 2005, 4280-4290.

(8) Zhang, L.; Ge, Y. Y.; Peng, F.; Du, M. Inorg. Chem. Commun. 2006, 9, 486-488.

(9) Manna, S. C.; Zangrando, E.; Drew, M. G. B.; Ribas, J.; Chaudhuri, N. R. Eur. J. Inorg. Chem. 2006, 481-490.

(10) Tsao, C. P.; Sheu, C. Y.; Nguyen, N.; Lii, K. H. Inorg. Chem. 2006, $45,6361-6364$.

6502 Inorganic Chemistry, Vol. 46, No. 16, 2007 these materials find interesting potential applications arising from their peculiar architectures such as zeolite-like frameworks ${ }^{13}$ and the inherent properties of the metallic centers, for example, photoluminescence arising from the presence of lanthanides. ${ }^{14}$

Following our ongoing research on crystalline the hybrid materials, ${ }^{15-25}$ we recently focused our attention on the use

(11) Mohanu, A.; Brouca-Cabarrecq, C.; Trombe, J. C. J. Solid State Chem. 2006, 179, 3-17.

(12) Chapelet-Arab, B.; Nowogrocki, G.; Abraham, F.; Grandjean, S. $J$. Solid State Chem. 2005, 178, 3055-3065.

(13) Mahe, N.; Audebrand, N. Solid State Sci. 2006, 8, 988-999.

(14) Song, J. L.; Mao, J. G. Chem. Eur. J. 2005, 11, 1417-1424.

(15) Soares-Santos, P. C. R.; Paz, F. A. A.; Ferreira, R. A. S.; Klinowski, J.; Carlos, L. D.; Trindade, T.; Nogueira, H. I. S. Polyhedron 2006, $25,2471-2482$.

(16) Shi, F. N.; Paz, F. A. A.; Girginova, P.; Rocha, J.; Amaral, V. S.; Klinowski, J.; Trindade, T. J. Mol. Struct. 2006, 789, 200-208.

(17) Paz, F. A. A.; Rocha, J.; Klinowski, J.; Trindade, T.; Shi, F. N.; Mafra, L. Prog. Solid State Chem. 2005, 33, 113-125.

(18) Shi, F. N.; Paz, F. A. A.; Girginova, P. I.; Nogueira, H. I. S.; Rocha, J.; Amaral, V. S.; Klinowski, J.; Trindade, T. J. Solid State Chem. 2006, 179, 1497-1505.

(19) Shi, F. N.; Paz, F. A. A.; Girginova, P. I.; Amaral, V. S.; Rocha, J.; Klinowski, J.; Trindade, T. Inorg. Chim. Acta 2006, 359, 1147-1158.

(20) Mafra, L.; Paz, F. A. A.; Shi, F. N.; Fernandez, C.; Trindade, T.; Klinowski, J.; Rocha, J. Inorg. Chem. Commun. 2006, 9, 34-38.

(21) Shi, F. N.; Paz, F. A. A.; Girginova, P. I.; Mafra, L.; Amaral, V. S.; Rocha, J.; Makal, A.; Wozniak, K.; Klinowski, J.; Trindade, T. J. Mol. Struct. 2005, 754, 51-60.

10.1021/ic700507j CCC: $\$ 37.00 \quad$ C 2007 American Chemical Society Published on Web 07/10/2007 
Scheme 1

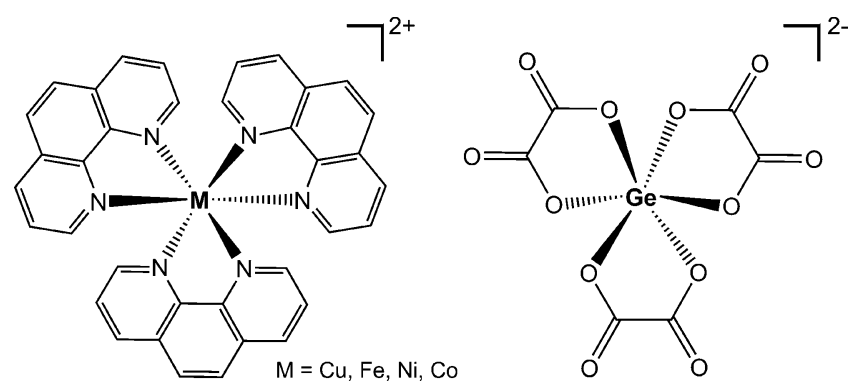

of germanium centers. ${ }^{26,27}$ These often exhibit two distinct oxidation states, +2 and +4 , with the latter being the most stable at ambient conditions and commonly appearing in inorganic compounds (such as $\mathrm{GeO}_{2}$ ) and germanate frameworks. $^{28,29}$ In addition, $\mathrm{Ge}^{4+}$ centers exhibit a number of distinct coordination numbers and environments, namely, four (often tetrahedral), ${ }^{30-32}$ five (square pyramidal or trigonal bipyramidal), ${ }^{31,33-36}$ and six (often octahedral), ${ }^{37,38}$ a crucial feature in order to achieve topological diversity for the frameworks.

Here we wish to report the first complex-based heterodimetallic crystalline compounds containing the divalent tris(oxalato- $O, O^{\prime}$ )germanate anion crystallizing with a number of transition-metal $(\mathrm{M})$ complexes with $1,10^{\prime}$-phenanthroline (phen) organic residues, $\left[\mathrm{M}(\mathrm{phen})_{3}\right]\left[\mathrm{Ge}\left(\mathrm{C}_{2} \mathrm{O}_{4}\right)_{3}\right] \cdot x \mathrm{H}_{2} \mathrm{O}$ [where $\mathrm{M}^{2+}=\mathrm{Cu}^{2+}(\mathbf{1} \mathbf{a}$ and $\mathbf{1 b}), \mathrm{Fe}^{2+}(\mathbf{2} \mathbf{a}$ and $\mathbf{2 b}), \mathrm{Ni}^{2+}(\mathbf{3}), \mathrm{Co}^{2+}$ (4); $x=0.2$ for $\mathbf{2 b}$ (Scheme 1). Furthermore, the first examples of binuclear complexes containing the neutral bis(oxalate- $\left.O, O^{\prime}\right)$ germanium fragment $\left[\mathrm{Ge}\left(\mathrm{C}_{2} \mathrm{O}_{4}\right)_{2}\right]$ are reported,

(22) Shi, F. N.; Paz, F. A. A.; Rocha, J.; Klinowski, J.; Trindade, T. Inorg. Chim. Acta 2005, 358, 927-932.

(23) Girginova, P. I.; Paz, F. A. A.; Nogueira, H. I. S.; Silva, N. J. O.; Amaral, V. S.; Klinowski, J.; Trindade, T. Polyhedron 2005, 24, 563569.

(24) Paz, F. A. A.; Klinowski, J. J. Solid State Chem. 2004, 177, $3423-$ 3432 .

(25) Paz, F. A. A.; Shi, F. N.; Klinowski, J.; Rocha, J.; Trindade, T. Eur. J. Inorg. Chem. 2004, 2759-2768.

(26) Mafra, L.; Paz, F. A. A.; Shi, F. N.; Rocha, J.; Trindade, T.; Fernandez, C.; Makal, A.; Wozniak, K.; Klinowski, J. Chem. Eur. J. 2005, 12, 363-375.

(27) Mafra, L.; Almeida Paz, F. A.; Shi, F. N.; Sá Ferreira, R. A.; Carlos, L. D.; Trindade, T.; Fernandez, C.; Klinowski, J.; Rocha, J. Eur. J. Inorg. Chem. 2006, 47-41-4751.

(28) Greenwood, N. N.; Earnshaw, A. Chem. Elements, 2nd ed.; Butterworth-Heinemann: Oxford, U.K., 1997.

(29) Cotton, F. A.; Wilkinson, G. Advanced Inorganic Chemistry, 5th ed.; John Wiley \& Sons: New York, 1988.

(30) Karlov, S. S.; Lermontova, E. K.; Zabalov, M. V.; Selina, A. A.; Churakov, A. V.; Howard, J. A. K.; Antipin, M. Y.; Zaitseva, G. S. Inorg. Chem. 2005, 44, 4879-4886.

(31) Schnepf, A. Eur. J. Inorg. Chem. 2005, 2120-2123.

(32) Iwamoto, T.; Masuda, H.; Ishida, S.; Kabuto, C.; Kira, M. J. Am. Chem. Soc. 2003, 125, 9300-9301.

(33) Chen, L.; Chen, J. X.; Sun, L. J.; Xie, Q. L. Appl. Organomet. Chem. 2005, 19, 1038-1042.

(34) Khrustalev, V. N.; Portnyagin, I. A.; Borisova, I. V.; Zemlyansky, N. N.; Ustynyuk, Y. A.; Antipin, M. Y.; Nechaev, M. S. Organometallics 2006, 25, 2501-2504.

(35) Takeuchi, Y.; Tanaka, K.; Tanaka, K.; Ohnishi-Kameyama, M.; Kalman, A.; Parkanyi, L. Chem. Commun. 1998, 2289-2290.

(36) Holmes, R. R.; Day, R. O.; Sau, A. C.; Poutasse, C. A.; Holmes, J. M. Inorg. Chem. 1985, 24, 193-199.

(37) Shen, X.; Nakashima, A.; Sakata, K.; Hashimoto, M. Inorg. Chem. Commun. 2004, 7, 621-624.

(38) Krivokapic, A.; Anderson, H. L.; Bourhill, G.; Ives, R.; Clark, S.; McEwan, K. J. Adv. Mater. 2001, 13, 652-656.

\section{Scheme 2}

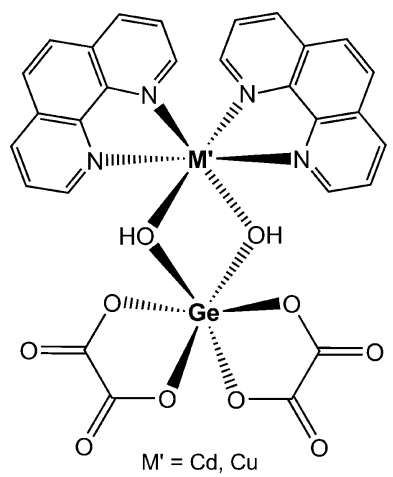

which is instead connected by two $\mu_{2}$-bridging hydroxyl groups to a cationic $\left[\mathrm{M}(\text { phen })_{2}\right]^{2+}$ fragment, $\left[\mathrm{MGe}(\mathrm{phen})_{2^{-}}\right.$ $\left(\mu_{2}-\mathrm{OH}\right)_{2}\left(\mathrm{C}_{2} \mathrm{O}_{4}\right)_{2}$ ] [where $\mathrm{M}^{2+}=\mathrm{Cd}^{2+}(\mathbf{5})$ and $\mathrm{Cu}^{2+}(\mathbf{6})$ ] (Scheme 2). For the former series of compounds, two polymorphs for $\mathrm{Cu}^{2+}$, and a pseudo-polymorph for $\mathrm{Fe}^{2+}$ could be isolated by employing slightly different synthetic procedures. Remarkably, searches in the literature and the Cambridge Structure Database (CSD, Version 5.28 Nov $2006)^{39,40}$ reveal that only a handful of structures containing $\left[\mathrm{Ge}\left(\mathrm{C}_{2} \mathrm{O}_{4}\right)_{3-x}\right]^{2(x-1)}$ moieties are known to date, all comprising small organic molecules or $\mathrm{K}^{+}$as the counterions. ${ }^{41-44}$

\section{Experimental Section}

General. Chemicals were readily available from commercial sources and used as received without further purification: amorphous germanium(IV) oxide $\left(\mathrm{GeO}_{2}, 99.99+\%\right.$, Aldrich), oxalic acid dihydrate $\left(\mathrm{H}_{2} \mathrm{C}_{2} \mathrm{O}_{4} \cdot 2 \mathrm{H}_{2} \mathrm{O}\right.$, $\geq 99 \%$, Panreac $), 1,10^{\prime}$-phenanthroline monohydrate (phen, $\mathrm{C}_{12} \mathrm{H}_{8} \mathrm{~N}_{2} \cdot \mathrm{H}_{2} \mathrm{O}, \geq 99.0 \%$, Fluka), copper(II) acetate monohydrate $\left(\mathrm{CuC}_{4} \mathrm{H}_{6} \mathrm{O}_{4} \cdot \mathrm{H}_{2} \mathrm{O}, 98 \%\right.$, Panreac), nickel(II) acetate tetrahydrate $\left(\mathrm{NiC}_{4} \mathrm{H}_{6} \mathrm{O}_{4} \cdot 4 \mathrm{H}_{2} \mathrm{O}, \geq 99 \%\right.$, Riedel-deHaën), cobalt(II) acetate tetrahydrate $\left(\mathrm{CoC}_{4} \mathrm{H}_{6} \mathrm{O}_{4} \cdot 4 \mathrm{H}_{2} \mathrm{O}, 98 \%\right.$, Panreac), cadmium(II) acetate dihydrate $\left(\mathrm{CdC}_{4} \mathrm{H}_{6} \mathrm{O}_{4} \cdot 2 \mathrm{H}_{2} \mathrm{O}, 98 \%\right.$, Fluka), ammonium ferrous sulfate hexahydrate $\left[\left(\mathrm{NH}_{4}\right)_{2} \mathrm{Fe}\left(\mathrm{SO}_{4}\right)_{2} \cdot 6 \mathrm{H}_{2} \mathrm{O}\right.$, $99 \%$, Merck], and potassium ferric oxalate $\left[\mathrm{KFe}\left(\mathrm{C}_{2} \mathrm{O}_{4}\right)_{2}, 99 \%\right.$, Ventron].

Instrumentation. Elemental analyses for $\mathrm{C}, \mathrm{H}$, and $\mathrm{N}$ were performed in a CHNS-932 elemental analyzer in the Microanalysis Laboratory of the University of Aveiro.

Thermogravimetric analyses (TGA) were carried out using a Shimadzu TGA 50 with a heating rate of $5{ }^{\circ} \mathrm{C} / \mathrm{min}$ under a continuous flow of air with rate of $20 \mathrm{~cm}^{3} / \mathrm{min}$.

Scanning electron microscopy (SEM) and energy dispersive analysis of X-rays spectroscopy (EDS) were performed using a Hitachi S-4100 field emission gun tungsten filament instrument working at $25 \mathrm{kV}$. Samples were prepared by deposition on aluminum sample holders and were carbon coated.

FT-IR spectra were collected from $\mathrm{KBr}$ pellets (Aldrich, 99\%+, FT-IR grade) on a Mattson 7000 FT-IR spectrometer. Fourier Transform Raman (FT-Raman) spectra (range 4000-100 $\mathrm{cm}^{-1}$ )

(39) Allen, F. H. Acta Crystallogr., Sect. B 2002, 58, 380-388.

(40) Allen, F. H.; Motherwell, W. D. S. Acta Crystallogr., Sect. B 2002, $58,407-422$

(41) Seiler, O.; Burschka, C.; Penka, M.; Tacke, R. Z. Anorg. Allg. Chem. 2002, 628, 2427-2434.

(42) Dean, P. A. W.; Dance, I. G.; Craig, D. C.; Scudder, M. L. Acta Crystallogr., Sect. C 2001, 57, 1030-1031.

(43) Martin, L.; Turner, S. S.; Day, P.; Guionneau, P.; Howard, J. A. K.; Uruichi, M.; Yakushi, K. J. Mater. Chem. 1999, 9, 2731-2736.

(44) Jorgensen, N.; Weakley, T. J. R. Dalton Trans. 1980, 2051-2052. 
were recorded on a Bruker RFS 100 spectrometer with a Nd:YAG coherent laser $(\lambda=1064 \mathrm{~nm})$.

Synthesis of $\left[\mathrm{Cu}(\text { phen })_{3}\right]\left[\mathrm{Ge}\left(\mathrm{C}_{2} \mathrm{O}_{4}\right)_{3}\right]$ (1a). A mixture containing $0.06 \mathrm{~g}$ of $\mathrm{GeO}_{2}, 0.18 \mathrm{~g}$ of $\mathrm{H}_{2} \mathrm{C}_{2} \mathrm{O}_{4} \cdot 2 \mathrm{H}_{2} \mathrm{O}, 0.10 \mathrm{~g}$ of $\mathrm{CuC}_{4} \mathrm{H}_{6} \mathrm{O}_{4} \cdot$ $\mathrm{H}_{2} \mathrm{O}$, and $0.20 \mathrm{~g}$ of phen was mixed in ca. $15 \mathrm{~g}$ of distilled water and stirred at ambient temperature for $30 \mathrm{~min}$. The resulting homogeneous suspension, with approximate molar composition of 1:3:1:2, respectively, was transferred to an autoclave (ca. $40 \mathrm{~mL}$ ) and heated at $100{ }^{\circ} \mathrm{C}$ for $72 \mathrm{~h}$. Large single crystals of $\left[\mathrm{Cu}(\text { phen })_{3}\right]-$ $\left[\mathrm{Ge}\left(\mathrm{C}_{2} \mathrm{O}_{4}\right)_{3}\right]$ were directly obtained from the autoclave contents along with some small impurities (later identified as compound $\mathbf{6}$, see below) which could not be eliminated by physical separation. The sample was washed with copious amounts of distilled water, filtrated, and air-dried under ambient conditions (yield $49.0 \%$ based on $\mathrm{CuC}_{4} \mathrm{H}_{6} \mathrm{O}_{4} \cdot \mathrm{H}_{2} \mathrm{O}$ ).

Calculated elemental composition (based on single-crystal data for $\mathrm{C}_{42} \mathrm{H}_{24} \mathrm{~N}_{6} \mathrm{O}_{12} \mathrm{CuGe}$, MW 940.80; \%): C 53.62, N 8.93, H 2.57. Found for the as-synthesized bulk material (\%): C 49.49, $\mathrm{N} 8.39, \mathrm{H}$ 2.41. TGA data (weight losses inside parentheses): $205-341{ }^{\circ} \mathrm{C}(-40.7 \%) ; 341-599{ }^{\circ} \mathrm{C}(-35.3 \%)$. Selected FT-IR and Raman (inside parentheses and in italics) data (in $\mathrm{cm}^{-1}$ ): $v(\mathrm{C}-\mathrm{H}$, aromatic $)=3068 \mathrm{w}, 2933 \mathrm{w},(3073) ; v($ uncoordinated carbonyl groups $)=1735 \mathrm{~s} ; v_{\text {asym }}\left(-\mathrm{CO}_{2}^{-}\right)=1676 \mathrm{~s}, 1627 \mathrm{~m}, 1588 \mathrm{w}$, $(1606,1545) ; v(\mathrm{C}-\mathrm{C}$, skeletal vibrations $)=1518 \mathrm{~m}, 1497 \mathrm{w},(1446)$; $\delta(\mathrm{C}-\mathrm{H})=1428 \mathrm{~s},(1411) ; v_{\mathrm{sym}}\left(-\mathrm{CO}_{2}^{-}\right)=1428 \mathrm{~s}, 1362 \mathrm{~s}, 1330 \mathrm{~s}$, $(1411) ; v(\mathrm{C}-\mathrm{N}$, heteroaromatic amines $)=1330 \mathrm{~s} ; v(\mathrm{C}-\mathrm{O})=$ 1226m, 1198w, 1146w, (1296); $v(\mathrm{C}-\mathrm{C}$ skeletal stretching mode $)$ $=1105 \mathrm{w},(1054) ; v(\mathrm{C}-\mathrm{H})=992 \mathrm{w} ; v(\mathrm{C}-\mathrm{C}$ and $\mathrm{Ge}-\mathrm{O})=893 \mathrm{w}$, $845 \mathrm{~m}, 819 \mathrm{~s},(807) ; \delta\left(\mathrm{CO}_{2}^{-}\right)=775 \mathrm{w}, 725 \mathrm{~s},(721) ; \gamma(\mathrm{C}-\mathrm{H})=$ $644 \mathrm{w}, 595 \mathrm{w} ; v(\mathrm{M}-\mathrm{N})$ and $\rho\left(\mathrm{CO}_{2}^{-}\right)=470 \mathrm{~m}, 426 \mathrm{w},(427)$.

Synthesis of $\left[\mathrm{Cu}(\text { phen })_{3}\right]\left[\mathrm{Ge}\left(\mathrm{C}_{2} \mathrm{O}_{4}\right)_{3}\right](\mathbf{1 b})$. The synthetic procedure used to isolate this polymorph is identical to that described for 1a but using instead a reaction mixture containing $0.050 \mathrm{~g}$ of $\mathrm{GeO}_{2}, 0.180 \mathrm{~g}$ of $\mathrm{H}_{2} \mathrm{C}_{2} \mathrm{O}_{4} \cdot 2 \mathrm{H}_{2} \mathrm{O}, 0.095 \mathrm{~g}$ of $\mathrm{CuC}_{4} \mathrm{H}_{6} \mathrm{O}_{4} \cdot \mathrm{H}_{2} \mathrm{O}$, $0.300 \mathrm{~g}$ of phen, and $15 \mathrm{~g}$ of $\mathrm{H}_{2} \mathrm{O}$ (approximate molar composition of $1 \mathrm{GeO}_{2}: 3 \mathrm{H}_{2} \mathrm{C}_{2} \mathrm{O}_{4}: 1 \mathrm{CuC}_{4} \mathrm{H}_{6} \mathrm{O}_{4}: 3$ phen). Yield $95.6 \%$ based on $\mathrm{CuC}_{4} \mathrm{H}_{6} \mathrm{O}_{4} \cdot \mathrm{H}_{2} \mathrm{O}$.

Calculated elemental composition (based on single-crystal data for $\mathrm{C}_{42} \mathrm{H}_{24} \mathrm{~N}_{6} \mathrm{O}_{12} \mathrm{CuGe}$, MW 940.80; \%): C 53.62, N 8.93, H 2.57. Found (\%): C 53.50, N 8.72, H 2.54. TGA data (weight losses inside parentheses): $201-343{ }^{\circ} \mathrm{C}(-48.0 \%) ; 343-599{ }^{\circ} \mathrm{C}(-31.9 \%)$. Selected FT-IR and Raman (inside parentheses and in italics) data (in $\left.\mathrm{cm}^{-1}\right): v(\mathrm{C}-\mathrm{H}$, aromatic) $=3059 \mathrm{w},(3069) ; v$ (uncoordinated carbonyl groups $)=1737 \mathrm{~s} ; v_{\text {asym }}\left(-\mathrm{CO}_{2}^{-}\right)=1669 \mathrm{w}, 1621 \mathrm{w}, 1588 \mathrm{w}$, $(1624,1605,1586) ; v(\mathrm{C}-\mathrm{C}$, skeletal vibrations $)=1515 \mathrm{~m}, 1493 \mathrm{w}$, $(1449) ; \delta(\mathrm{C}-\mathrm{H})=1425 \mathrm{~m},(1416) ; v_{\mathrm{sym}}\left(-\mathrm{CO}_{2}^{-}\right)=1425 \mathrm{~m}, 1318 \mathrm{~s}$, $(1416,1307) ; v(\mathrm{C}-\mathrm{N}$, heteroaromatic amines $)=1318 \mathrm{~s},(1307)$; $v(\mathrm{C}-\mathrm{O})=1225 \mathrm{~m}, 1191 \mathrm{w}, 1139 \mathrm{w} ; v(\mathrm{C}-\mathrm{C}$ skeletal stretching mode $)=1102 \mathrm{w},(1053) ; v(\mathrm{C}-\mathrm{H})=1000 \mathrm{w}, 965 \mathrm{w} ; v(\mathrm{C}-\mathrm{C}$ and $\mathrm{Ge}-\mathrm{O})=892 \mathrm{~m}, 853 \mathrm{~m}, 821 \mathrm{~s} ; \delta\left(\mathrm{CO}_{2}{ }^{-}\right)=778 \mathrm{w}, 724 \mathrm{~m}$, (735); $\gamma(\mathrm{C}-\mathrm{H})=645 \mathrm{w}, 593 \mathrm{w} ; \nu(\mathrm{M}-\mathrm{N})$ and $\rho\left(\mathrm{CO}_{2}^{-}\right)=470 \mathrm{~m}, 420 \mathrm{w}$, (425).

Synthesis of $\left[\mathrm{Fe}(\text { phen })_{3}\right]\left[\mathrm{Ge}\left(\mathrm{C}_{2} \mathrm{O}_{4}\right)_{3}\right](2 \mathrm{a})$. The synthetic procedure used to isolate $\left[\mathrm{Fe}(\text { phen })_{3}\right]\left[\mathrm{Ge}\left(\mathrm{C}_{2} \mathrm{O}_{4}\right)_{3}\right]$ is identical to that described for 1a with the reaction mixture containing instead $0.050 \mathrm{~g}$ of $\mathrm{GeO}_{2}, 0.140 \mathrm{~g}$ of $\mathrm{H}_{2} \mathrm{C}_{2} \mathrm{O}_{4} \cdot 2 \mathrm{H}_{2} \mathrm{O}, 0.110 \mathrm{~g}$ of $\mathrm{KFe}\left(\mathrm{C}_{2} \mathrm{O}_{4}\right)_{2}$, $0.200 \mathrm{~g}$ of phen, and $15 \mathrm{~g}$ of $\mathrm{H}_{2} \mathrm{O}$ (approximate molar composition of $1 \mathrm{GeO}_{2}: 2 \mathrm{H}_{2} \mathrm{C}_{2} \mathrm{O}_{4}: 2 / 3 \mathrm{KFe}\left(\mathrm{C}_{2} \mathrm{O}_{4}\right)_{2}: 2$ phen $)$. Yield $58.1 \%$ based on $\mathrm{KFe}\left(\mathrm{C}_{2} \mathrm{O}_{4}\right)_{2}$.

Calculated elemental composition (based on single-crystal data for $\mathrm{C}_{42} \mathrm{H}_{24} \mathrm{~N}_{6} \mathrm{O}_{12} \mathrm{FeGe}$, MW 933.11; \%): C 54.06, N 9.01, H 2.59. Found (\%): C 53.70, N 8.76, H 2.49. TGA data (weight losses inside parentheses): $277-353{ }^{\circ} \mathrm{C}(-59.0 \%) ; 353-598^{\circ} \mathrm{C}(-21.0 \%)$.
Selected FT-IR and Raman (inside parentheses and in italics) data (in $\left.\mathrm{cm}^{-1}\right): v(\mathrm{C}-\mathrm{H}$, aromatic) $=3076 \mathrm{w},(3076) ; v($ uncoordinated carbonyl groups $)=1734 \mathrm{~s},(1763) ; v_{\text {asym }}\left(-\mathrm{CO}_{2}^{-}\right)=1671 \mathrm{~m}, 1631 \mathrm{w}$, $1577 \mathrm{w},(1632,1602,1580) ; v(\mathrm{C}-\mathrm{C}$, skeletal vibrations $)=1512 \mathrm{w}$, $1494 \mathrm{w},(1513,1454) ; \delta(\mathrm{C}-\mathrm{H})=1425 \mathrm{~s},(1431) ; v_{\mathrm{sym}}\left(-\mathrm{CO}_{2}^{-}\right)=$ $1425 \mathrm{~s}, 1363 \mathrm{w}, 1328 \mathrm{~s},(1431,1345,1304) ; v(\mathrm{C}-\mathrm{N}$, heteroaromatic amines $)=1328 \mathrm{~s},(1304) ; v(\mathrm{C}-\mathrm{O})=1227 \mathrm{~s}, 1197 \mathrm{~m}, 1140 \mathrm{w},(1208$, $1142,1105) ; v(\mathrm{C}-\mathrm{C}$ skeletal stretching mode $)=1096 \mathrm{w}, 1054 \mathrm{w}$, (1055); $v(\mathrm{C}-\mathrm{H})=987 \mathrm{w},(911) ; v(\mathrm{C}-\mathrm{C}$ and $\mathrm{Ge}-\mathrm{O})=892 \mathrm{~m}, 842 \mathrm{~s}$, $819 \mathrm{~s},(877) ; \delta\left(\mathrm{CO}_{2}^{-}\right)=771 \mathrm{w}, 721 \mathrm{~s},(739) ; \gamma(\mathrm{C}-\mathrm{H})=645 \mathrm{w}, 595 \mathrm{w}$, $532 \mathrm{w},(646) ; \nu(\mathrm{M}-\mathrm{N})$ and $\rho\left(\mathrm{CO}_{2}^{-}\right)=470 \mathrm{~m},(439)$.

Synthesis of $\left[\mathrm{Fe}(\mathrm{phen})_{3}\right]\left[\mathrm{Ge}\left(\mathrm{C}_{2} \mathrm{O}_{4}\right)_{3}\right] \cdot \mathbf{0 . 2}_{2} \mathrm{O}(2 \mathrm{~b})$. The synthetic procedure used to isolate this hydrated polymorph of $[\mathrm{Fe}-$ (phen $\left.)_{3}\right]\left[\mathrm{Ge}\left(\mathrm{C}_{2} \mathrm{O}_{4}\right)_{3}\right]$ is identical to that described for $\mathbf{1 a}$ but using instead $\left(\mathrm{NH}_{4}\right)_{2} \mathrm{Fe}\left(\mathrm{SO}_{4}\right)_{2} \cdot 6 \mathrm{H}_{2} \mathrm{O}$ as the $\mathrm{Fe}^{2+}$ source. The reaction mixture contained $0.050 \mathrm{~g}$ of $\mathrm{GeO}_{2}, 0.180 \mathrm{~g}$ of $\mathrm{H}_{2} \mathrm{C}_{2} \mathrm{O}_{4} \cdot 2 \mathrm{H}_{2} \mathrm{O}$, $0.130 \mathrm{~g}$ of $\left(\mathrm{NH}_{4}\right)_{2} \mathrm{Fe}\left(\mathrm{SO}_{4}\right)_{2} \cdot 6 \mathrm{H}_{2} \mathrm{O}, 0.200 \mathrm{~g}$ of phen, and $15 \mathrm{~g}$ of $\mathrm{H}_{2} \mathrm{O}$ (approximate molar composition of $1 \mathrm{GeO}_{2}: 3 \mathrm{H}_{2} \mathrm{C}_{2} \mathrm{O}_{4}: 2 / 3\left(\mathrm{NH}_{4}\right)_{2}-$ $\mathrm{Fe}\left(\mathrm{SO}_{4}\right)_{2} \cdot 6 \mathrm{H}_{2} \mathrm{O}$ :2phen). Yield $53.5 \%$ based on $\left(\mathrm{NH}_{4}\right)_{2} \mathrm{Fe}\left(\mathrm{SO}_{4}\right)_{2}$. $6 \mathrm{H}_{2} \mathrm{O}$.

Calculated elemental composition (based on single-crystal data for $\mathrm{C}_{42} \mathrm{H}_{24.40} \mathrm{~N}_{6} \mathrm{O}_{12.20} \mathrm{FeGe}$, MW 936.72; \%): C 53.85, N 8.97, $\mathrm{H}$ 2.63. Found (\%): C 53.12, N 8.68, H 2.64. TGA data (weight losses inside parentheses): $30-268^{\circ} \mathrm{C}(-0.8 \%) ; 268-342{ }^{\circ} \mathrm{C}(-59.8 \%)$; 342-599 ${ }^{\circ} \mathrm{C}(-18.8 \%)$. Selected FT-IR and Raman (inside parentheses and in italics) data (in $\left.\mathrm{cm}^{-1}\right): v(\mathrm{C}-\mathrm{H}$, aromatic) $=$ $3071 \mathrm{~m},(3071)$; $v$ (uncoordinated carbonyl groups $)=1740 \mathrm{~s},(1765$, $1727) ; \quad v_{\text {asym }}\left(-\mathrm{CO}_{2}^{-}\right)=1672 \mathrm{~s}, 1614 \mathrm{~s},(1630,1600,1580)$; $v(\mathrm{C}-\mathrm{C}$, skeletal vibrations $)=1517 \mathrm{~m}, 1494 \mathrm{w},(1513,1453)$; $\delta(\mathrm{C}-\mathrm{H})=1424 \mathrm{~s},(1427) ; v_{\mathrm{sym}}\left(-\mathrm{CO}_{2}^{-}\right)=1424 \mathrm{~s}, 1358 \mathrm{~m}, 1313 \mathrm{~s}$, (1427, 1341, 1299); $v(\mathrm{C}-\mathrm{N}$, heteroaromatic amines $)=1313 \mathrm{~s}$, (1299); $v(\mathrm{C}-\mathrm{O})=1218 \mathrm{~m}, 1186 \mathrm{w}, 1141 \mathrm{w},(1206,1141) ; v(\mathrm{C}-\mathrm{C}$ skeletal stretching mode $)=1105 \mathrm{w},(1106,1056) ; v(\mathrm{C}-\mathrm{C}$ and $\mathrm{Ge}-$ O) $=893 \mathrm{~m}, 849 \mathrm{~m}, 817 \mathrm{~s},(875) ; \delta\left(\mathrm{CO}_{2}^{-}\right)=725 \mathrm{~s},(741) ; \gamma(\mathrm{C}-\mathrm{H})$ $=646 \mathrm{w}, 596 \mathrm{w}, 530 \mathrm{w},(647,596) ; v(\mathrm{M}-\mathrm{N})$ and $\rho\left(\mathrm{CO}_{2}^{-}\right)=470 \mathrm{~m}$, 420w, (437).

Synthesis of $\left[\mathrm{Ni}(\text { phen })_{3}\right]\left[\mathrm{Ge}\left(\mathrm{C}_{2} \mathrm{O}_{4}\right)_{3}\right](3)$. The synthetic procedure used to isolate $\left[\mathrm{Ni}(\text { phen })_{3}\right]\left[\mathrm{Ge}\left(\mathrm{C}_{2} \mathrm{O}_{4}\right)_{3}\right]$ is identical to that described for $1 \mathrm{a}$ but using instead $\mathrm{NiC}_{4} \mathrm{H}_{6} \mathrm{O}_{4} \cdot 4 \mathrm{H}_{2} \mathrm{O}$ as the $\mathrm{Ni}^{2+}$ source. The reaction mixture contained $0.050 \mathrm{~g}$ of $\mathrm{GeO}_{2}, 0.180 \mathrm{~g}$ of $\mathrm{H}_{2} \mathrm{C}_{2} \mathrm{O}_{4} \cdot 2 \mathrm{H}_{2} \mathrm{O}, 0.080 \mathrm{~g}$ of $\mathrm{NiC}_{4} \mathrm{H}_{6} \mathrm{O}_{4} \cdot 4 \mathrm{H}_{2} \mathrm{O}, 0.200 \mathrm{~g}$ of phen, and $15 \mathrm{~g}$ of $\mathrm{H}_{2} \mathrm{O}$ (approximate molar composition of $1 \mathrm{GeO}_{2}$ : $3 \mathrm{H}_{2} \mathrm{C}_{2} \mathrm{O}_{4}: 2 / 3 \mathrm{NiC}_{4} \mathrm{H}_{6} \mathrm{O}_{4}: 2$ phen). Yield $53.5 \%$ based on $\mathrm{NiC}_{4} \mathrm{H}_{6} \mathrm{O}_{4}$. $4 \mathrm{H}_{2} \mathrm{O}$.

Calculated elemental composition (based on single-crystal data for $\mathrm{C}_{42} \mathrm{H}_{24} \mathrm{~N}_{6} \mathrm{O}_{12} \mathrm{NiGe}$, MW 935.97; \%): C 53.90, N 8.98, H 2.58. Found (\%): C 52.98, N 9.01, H 2.55. TGA data (weight losses inside parentheses): $280-374{ }^{\circ} \mathrm{C}(-44.9 \%) ; 374-599^{\circ} \mathrm{C}(-34.5 \%)$. Selected FT-IR and Raman (inside parentheses and in italics) data (in $\left.\mathrm{cm}^{-1}\right): v(\mathrm{C}-\mathrm{H}$, aromatic) $=3073 \mathrm{w},(3079) ; v($ uncoordinated carbonyl groups $)=1737 \mathrm{~s},(1759,1721) ; v_{\text {asym }}\left(-\mathrm{CO}_{2}^{-}\right)=1676 \mathrm{~s}$, $1627 \mathrm{~m}, 1588 \mathrm{w},(1627,1611,1588) ; v(\mathrm{C}-\mathrm{C}$, skeletal vibrations $)$ $=1518 \mathrm{~m}, 1497 \mathrm{w},(1517,1461) ; \delta(\mathrm{C}-\mathrm{H})=1428 \mathrm{~s},(1423)$; $v_{\text {sym }}\left(-\mathrm{CO}_{2}^{-}\right)=1428 \mathrm{~s}, 1362 \mathrm{~s}, 1330 \mathrm{~s},(1423,1362,1345,1322$, $1311) ; v(\mathrm{C}-\mathrm{N}$, heteroaromatic amines $)=1330 \mathrm{~s},(1322,1311)$; $v(\mathrm{C}-\mathrm{O})=1226 \mathrm{~m}, 1198 \mathrm{w}, 1146 \mathrm{w},(1258,1206,1146) ; v(\mathrm{C}-\mathrm{C}$ skeletal stretching mode $)=1105 \mathrm{w},(1056) ; v(\mathrm{C}-\mathrm{H})=992 \mathrm{w},(905)$; $v(\mathrm{C}-\mathrm{C}$ and $\mathrm{Ge}-\mathrm{O})=893 \mathrm{w}, 845 \mathrm{~m}, 819 \mathrm{~s},(872) ; \delta\left(\mathrm{CO}_{2}{ }^{-}\right)=775 \mathrm{w}$, $725 \mathrm{~s},(733) ; \gamma(\mathrm{C}-\mathrm{H})=644 \mathrm{w}, 595 \mathrm{w},(597,560) ; v(\mathrm{M}-\mathrm{N})$ and $\rho\left(\mathrm{CO}_{2}{ }^{-}\right)=470 \mathrm{~m}, 426 \mathrm{w},(514,485,426)$.

Synthesis of $\left[\mathrm{Co}(\text { phen })_{3}\right]\left[\mathrm{Ge}\left(\mathrm{C}_{2} \mathrm{O}_{4}\right)_{3}\right](4)$. The synthetic procedure used to isolate $\left[\mathrm{Co}(\text { phen })_{3}\right]\left[\mathrm{Ge}\left(\mathrm{C}_{2} \mathrm{O}_{4}\right)_{3}\right]$ as a microcrystalline light-yellow powder is identical to that described for $\mathbf{1 a}$ but using 
Table 1. Crystal Data Collection and Refinement Details for $\left[\mathrm{M}(\text { phen })_{3}\right]\left[\mathrm{Ge}\left(\mathrm{C}_{2} \mathrm{O}_{4}\right)_{3}\right] \cdot x \mathrm{H}_{2} \mathrm{O}$ [where $\mathrm{M}^{2+}=\mathrm{Cu}^{2+}(\mathbf{1 a}$ and $\mathbf{1 b}), \mathrm{Fe}^{2+}(\mathbf{2 a}$ and $\mathbf{2 b}), \mathrm{Ni}^{2+}$ $(3) ; x=0.2$ for $\mathbf{2 b}$ ] and $\left[\mathrm{MGe}(\text { phen })_{2}\left(\mu_{2}-\mathrm{OH}\right)_{2}\left(\mathrm{C}_{2} \mathrm{O}_{4}\right)_{2}\right]$ [where $\mathrm{M}^{2+}=\mathrm{Cd}^{2+}(\mathbf{5})$ and $\left.\mathrm{Cu}^{2+}(\mathbf{6})\right]$

\begin{tabular}{|c|c|c|c|c|}
\hline & $1 \mathbf{a}$ & 1b & $2 \mathbf{a}$ & $2 \mathbf{b}$ \\
\hline formula & $\mathrm{C}_{42} \mathrm{H}_{24} \mathrm{~N}_{6} \mathrm{O}_{12} \mathrm{CuGe}$ & $\mathrm{C}_{42} \mathrm{H}_{24} \mathrm{~N}_{6} \mathrm{O}_{12} \mathrm{CuGe}$ & $\mathrm{C}_{42} \mathrm{H}_{24} \mathrm{~N}_{6} \mathrm{O}_{12} \mathrm{FeGe}$ & $\mathrm{C}_{42} \mathrm{H}_{24.40} \mathrm{~N}_{6} \mathrm{O}_{12.20} \mathrm{FeGe}$ \\
\hline mol wt & 940.80 & 940.80 & 933.11 & 936.72 \\
\hline cryst description & blue-green prisms & blue-green prisms & red needles & red needles \\
\hline cryst size/mm & $0.28 \times 0.16 \times 0.15$ & $0.16 \times 0.15 \times 0.13$ & $0.32 \times 0.12 \times 0.10$ & $0.30 \times 0.07 \times 0.02$ \\
\hline temp $/ \mathrm{K}$ & $100(2)$ & $150(2)$ & $150(2)$ & $150(2)$ \\
\hline instrument & Smart 1000 & Bruker X8 & Bruker X8 & Bruker X8 \\
\hline cryst syst & monoclinic & monoclinic & monoclinic & triclinic \\
\hline space group & $P 2_{1} / n$ & $P 2_{1} / c$ & $P 2_{1} / n$ & $P \overline{1}$ \\
\hline$a / \AA$ & $9.4730(19)$ & $14.1076(9)$ & $9.3425(5)$ & $11.3137(5)$ \\
\hline$b / \AA$ & $20.528(4)$ & $12.9140(8)$ & $20.6117(9)$ & $12.0536(5)$ \\
\hline$c / \AA ̊$ & $19.029(4)$ & 20.8073(13) & 19.1881(9) & $16.0827(11)$ \\
\hline$\alpha / \mathrm{deg}$ & 90 & 90 & 90 & $109.059(4)$ \\
\hline$\beta / \mathrm{deg}$ & $96.19(3)$ & $97.982(3)$ & $95.410(2)$ & $94.664(4)$ \\
\hline$\gamma / \mathrm{deg}$ & 90 & 90 & 90 & 111.536 \\
\hline vol. $/ \AA^{3}$ & $3678.90(13)$ & $3754.1(4)$ & 3678.5 & 1877.10 \\
\hline $\mathrm{Z}$ & 4 & 4 & 4 & 2 \\
\hline$\rho_{\text {calcd }} / \mathrm{g} \mathrm{cm}^{-3}$ & 1.699 & 1.665 & 1.685 & 1.657 \\
\hline$F(000)$ & 1900 & 1900 & 1888 & 948 \\
\hline$\mu / \mathrm{mm}^{-1}$ & 1.475 & 1.445 & 1.291 & 1.266 \\
\hline$\theta$ range/deg & $3.68-28.40$ & $1.98-25.12$ & $1.45-30.21$ & $3.59-29.13$ \\
\hline \multirow[t]{3}{*}{ index ranges } & $-12 \leq h \leq 12$ & $-15 \leq h \leq 16$ & $-13 \leq h \leq 9$ & $-15 \leq h \leq 15$ \\
\hline & $-27 \leq k \leq 27$ & $-15 \leq k \leq 15$ & $-29 \leq k \leq 28$ & $-16 \leq k \leq 16$ \\
\hline & $-25 \leq l \leq 24$ & $-24 \leq l \leq 24$ & $-26 \leq l \leq 26$ & $-22 \leq l \leq 22$ \\
\hline reflns collected & 65748 & 66242 & 75853 & 32834 \\
\hline independent reflns & $9202\left(R_{\mathrm{int}}=0.0797\right)$ & $6659\left(R_{\mathrm{int}}=0.0699\right)$ & $10399\left(R_{\text {int }}=0.0400\right)$ & $9906\left(R_{\mathrm{int}}=0.0646\right)$ \\
\hline \multirow[t]{2}{*}{ final $R$ indices $[I>2 \sigma(I)]$} & $R_{1}=0.0386$ & $R_{1}=0.0309$ & $R_{1}=0.0335$ & $R_{1}=0.0418$ \\
\hline & $\mathrm{w} R_{2}=0.0721$ & $\mathrm{w} R_{2}=0.0672$ & $\mathrm{w} R_{2}=0.0793$ & $\mathrm{w} R_{2}=0.0826$ \\
\hline final $R$ indices (all data) & $R_{1}=0.0755$ & $R_{1}=0.0458$ & $R_{1}=0.0546$ & $R_{1}=0.0860$ \\
\hline & $\mathrm{w} R_{2}=0.0850$ & $\mathrm{w} R_{2}=0.0744$ & $\mathrm{w} R_{2}=0.0896$ & $\mathrm{w} R_{2}=0.0981$ \\
\hline \multirow[t]{2}{*}{ largest diff. peak and hole/e $\AA^{3}$} & 0.487 and -0.489 & 0.389 and -0.452 & 0.408 and -0.634 & 0.691 and -0.717 \\
\hline & \multicolumn{2}{|c|}{3} & 5 & 6 \\
\hline formula & \multicolumn{2}{|c|}{$\mathrm{C}_{42} \mathrm{H}_{24} \mathrm{~N}_{6} \mathrm{O}_{12} \mathrm{NiGe}$} & $\mathrm{C}_{28} \mathrm{H}_{18} \mathrm{~N}_{4} \mathrm{O}_{10} \mathrm{CdGe}$ & $\mathrm{C}_{28} \mathrm{H}_{18} \mathrm{~N}_{4} \mathrm{O}_{10} \mathrm{CuGe}$ \\
\hline mol wt & \multicolumn{2}{|c|}{935.97} & 755.45 & 706.59 \\
\hline cryst description & \multirow{2}{*}{\multicolumn{2}{|c|}{$\begin{array}{l}\text { pink prisms } \\
0.12 \times 0.05 \times 0.05\end{array}$}} & colorless prisms & green prisms \\
\hline cryst size/mm & & & $0.20 \times 0.12 \times 0.08$ & $0.31 \times 0.20 \times 0.18$ \\
\hline temp/K & \multicolumn{2}{|c|}{$\begin{array}{l}0.12 \times 0.05 \times 0.05 \\
110(2)\end{array}$} & $150(2)$ & $100(2)$ \\
\hline instrument & \multicolumn{2}{|c|}{ Kappa FR591 2000} & Bruker X8 & Smart 1000 \\
\hline cryst syst & \multirow{2}{*}{\multicolumn{2}{|c|}{$\begin{array}{l}\text { monoclinic } \\
P 2_{1} / n\end{array}$}} & monoclinic & orthorhombic \\
\hline space group & & & $C 2 / c$ & Pnna \\
\hline$a / \AA$ & \multicolumn{2}{|c|}{$9.4060(19)$} & $12.7304(2)$ & $15.250(3)$ \\
\hline$b / \AA$ & \multicolumn{2}{|c|}{$20.647(4)$} & $17.0619(3)$ & $12.032(2)$ \\
\hline$c / \AA ̊$ & $19.022(4)$ & & $12.1242(2)$ & $13.582(3)$ \\
\hline$\alpha / \operatorname{deg}$ & 90 & & 90 & 90 \\
\hline$\beta / \mathrm{deg}$ & $95.09(3)$ & & $100.729(1)$ & 90 \\
\hline$\gamma / \operatorname{deg}$ & 90 & & 90 & 90 \\
\hline vol. $/ \AA^{3}$ & $3679.6(13$ & & $2587.40(7)$ & 2492.1(9) \\
\hline $\mathrm{Z}$ & 4 & & 8 & 4 \\
\hline$\rho_{\text {calcd }} / \mathrm{g} \mathrm{cm}^{-3}$ & 1.690 & & 1.939 & 1.883 \\
\hline$F(000)$ & 1896 & & 1496 & 1420 \\
\hline$\mu / \mathrm{mm}^{-1}$ & 2.329 & & 2.056 & 2.134 \\
\hline$\theta$ range/deg & $3.83-27.5$ & & $3.70-27.48$ & $3.70-26.39$ \\
\hline index ranges & $-9 \leq h \leq$ & & $-16 \leq h \leq 16$ & $-19 \leq h \leq 15$ \\
\hline & $0 \leq k \leq 2$ & & $-22 \leq k \leq 22$ & $-15 \leq k \leq 15$ \\
\hline & $0 \leq l \leq 20$ & & $-15 \leq l \leq 15$ & $-16 \leq l \leq 16$ \\
\hline reflns collected & 21054 & & 41597 & 21786 \\
\hline independent reflns & $4523\left(R_{\text {int }}\right.$ & 531) & $2956\left(R_{\mathrm{int}}=0.0273\right)$ & $2557\left(R_{\mathrm{int}}=0.0975\right)$ \\
\hline final $R$ indices $[\mathrm{I}>2 \sigma(\mathrm{I})]$ & $R_{1}=0.05$ & & $R_{1}=0.0153$ & $R_{1}=0.1050$ \\
\hline & $\mathrm{w} R_{2}=0.1$ & & $\mathrm{w} R_{2}=0.0392$ & $\mathrm{w} R_{2}=0.2622$ \\
\hline final $R$ indices (all data) & $R_{1}=0.05$ & & $R_{1}=0.0176$ & $R_{1}=0.1387$ \\
\hline & $\mathrm{w} R_{2}=0.1$ & & $\mathrm{w} R_{2}=0.0401$ & $\mathrm{w} R_{2}=0.2798$ \\
\hline largest diff. peak and hole/e $\AA^{3}$ & 0.667 and & & 0.361 and -0.247 & 0.901 and -0.999 \\
\hline
\end{tabular}

instead $\mathrm{CoC}_{4} \mathrm{H}_{6} \mathrm{O}_{4} \cdot 4 \mathrm{H}_{2} \mathrm{O}$ as the $\mathrm{Co}^{2+}$ source. The reaction mixture contained $0.050 \mathrm{~g}$ of $\mathrm{GeO}_{2}, 0.180 \mathrm{~g}$ of $\mathrm{H}_{2} \mathrm{C}_{2} \mathrm{O}_{4} \cdot 2 \mathrm{H}_{2} \mathrm{O}, 0.080 \mathrm{~g}$ of $\mathrm{CoC}_{4} \mathrm{H}_{6} \mathrm{O}_{4} \cdot 4 \mathrm{H}_{2} \mathrm{O}, 0.200 \mathrm{~g}$ of phen, and $15 \mathrm{~g}$ of $\mathrm{H}_{2} \mathrm{O}$ (approximate molar composition of $1 \mathrm{GeO}_{2}: 3 \mathrm{H}_{2} \mathrm{C}_{2} \mathrm{O}_{4}: 2 / 3 \mathrm{CoC}_{4} \mathrm{H}_{6} \mathrm{O}_{4}: 2 \mathrm{phen}$ ). Yield $83.1 \%$ based on $\mathrm{CoC}_{4} \mathrm{H}_{6} \mathrm{O}_{4} \cdot 4 \mathrm{H}_{2} \mathrm{O}$.

Calculated elemental composition (for $\mathrm{C}_{42} \mathrm{H}_{24} \mathrm{~N}_{6} \mathrm{O}_{12} \mathrm{CoGe}$, MW 936.21; \%): C 53.88, N 8.98, H 2.58. Found (\%): C 52.00, N 8.63, $\mathrm{H}$ 2.54. TGA data (weight losses inside parentheses): $272-360{ }^{\circ} \mathrm{C}$ $(-46.7 \%) ; 360-598{ }^{\circ} \mathrm{C}(-33.3 \%)$. Selected FT-IR and Raman (inside parentheses and in italics) data $\left(\right.$ in $\left.^{-1}\right): v(\mathrm{C}-\mathrm{H}$, aromatic) $=3061 \mathrm{w}, 2925 \mathrm{w},(3081) ; v($ uncoordinated carbonyl groups $)=$ $1735 \mathrm{~s},(1763,1721) ; v_{\text {asym }}\left(-\mathrm{CO}_{2}^{-}\right)=1672 \mathrm{~m}, 1624 \mathrm{w}, 1605 \mathrm{w}$, $1583 \mathrm{w},(1626,1604,1586) ; v(\mathrm{C}-\mathrm{C}$, skeletal vibrations $)=1517 \mathrm{~m}$, $1496 \mathrm{w},(1517,1453) ; \delta(\mathrm{C}-\mathrm{H})=1426 \mathrm{~s},(1422) ; v_{\mathrm{sym}}\left(-\mathrm{CO}_{2}{ }^{-}\right)=$ $1426 \mathrm{~s}, 1316 \mathrm{~s},(1422,1362,1345,1306) ; v(\mathrm{C}-\mathrm{N}$, heteroaromatic amines $)=1316 \mathrm{~s},(1306) ; v(\mathrm{C}-\mathrm{O})=1227 \mathrm{~m}, 1197 \mathrm{w}, 1187 \mathrm{w}$, $1141 \mathrm{w},(1259,1145) ; v(\mathrm{C}-\mathrm{C}$ skeletal stretching mode $)=1104 \mathrm{w}$, $(1054) ; v(\mathrm{C}-\mathrm{H})=992 \mathrm{w}, 952 \mathrm{w},(901) ; v(\mathrm{C}-\mathrm{C}$ and $\mathrm{Ge}-\mathrm{O})=$ 
Table 2. Selected Bond Lengths $(\AA)$ for the Octahedral $\left\{\mathrm{MN}_{6}\right\}$ and $\left\{\mathrm{GeO}_{6}\right\}$ Coordination Environments Present in Compounds 1-3 $\left(\mathrm{M}^{2+}=\right.$ $\mathrm{Cu}^{2+}, \mathrm{Fe}^{2+}$, or $\mathrm{Ni}^{2+}$ )

\begin{tabular}{lccccc}
\hline & $\mathbf{1 a}\left(\mathrm{Cu}^{2+}\right)$ & $\mathbf{1 b}\left(\mathrm{Cu}^{2+}\right)$ & $\mathbf{2 a}\left(\mathrm{Fe}^{2+}\right)$ & $\mathbf{2 b}\left(\mathrm{Fe}^{2+}\right)$ & $\mathbf{3}\left(\mathrm{Ni}^{2+}\right)$ \\
\hline $\mathrm{M}(1)-\mathrm{N}(1)$ & $2.049(2)$ & $2.374(2)$ & $1.992(2)$ & $1.974(2)$ & $2.091(3)$ \\
$\mathrm{M}(1)-\mathrm{N}(2)$ & $2.025(2)$ & $2.031(2)$ & $1.974(2)$ & $1.986(2)$ & $2.081(3)$ \\
$\mathrm{M}(1)-\mathrm{N}(3)$ & $2.316(2)$ & $2.021(2)$ & $1.984(2)$ & $1.985(2)$ & $2.088(3)$ \\
$\mathrm{M}(1)-\mathrm{N}(4)$ & $2.059(2)$ & $2.317(2)$ & $1.978(2)$ & $1.985(2)$ & $2.076(3)$ \\
$\mathrm{M}(1)-\mathrm{N}(5)$ & $2.042(2)$ & $2.048(2)$ & $1.990(2)$ & $2.002(2)$ & $2.097(3)$ \\
$\mathrm{M}(1)-\mathrm{N}(6)$ & $2.275(2)$ & $2.056(2)$ & $1.978(1)$ & $1.972(2)$ & $2.085(3)$ \\
$\mathrm{Ge}(1)-\mathrm{O}(1)$ & $1.883(2)$ & $1.889(2)$ & $1.891(1)$ & $1.897(2)$ & $1.865(2)$ \\
$\mathrm{Ge}(1)-\mathrm{O}(3)$ & $1.886(2)$ & $1.872(2)$ & $1.892(1)$ & $1.894(2)$ & $1.880(2)$ \\
$\mathrm{Ge}(1)-\mathrm{O}(5)$ & $1.875(2)$ & $1.870(2)$ & $1.888(1)$ & $1.893(2)$ & $1.871(2)$ \\
$\mathrm{Ge}(1)-\mathrm{O}(7)$ & $1.876(2)$ & $1.879(2)$ & $1.878(1)$ & $1.890(2)$ & $1.879(2)$ \\
$\mathrm{Ge}(1)-\mathrm{O}(9)$ & $1.876(2)$ & $1.895(2)$ & $1.884(1)$ & $1.881(2)$ & $1.886(3)$ \\
$\mathrm{Ge}(1)-\mathrm{O}(11)$ & $1.870(2)$ & $1.888(2)$ & $1.878(1)$ & $1.890(2)$ & $1.881(2)$
\end{tabular}

892w, 867w, 845m, 819s, (869); $\delta\left(\mathrm{CO}_{2}^{-}\right)=772 \mathrm{w}, 725 \mathrm{~s},(733)$; $\gamma(\mathrm{C}-\mathrm{H})=644 \mathrm{w}, 602 \mathrm{w},(640,598,558) ; \nu(\mathrm{M}-\mathrm{N})$ and $\rho\left(\mathrm{CO}_{2}{ }^{-}\right)$ $=470 \mathrm{~m}, 425 \mathrm{w},(425)$.

Synthesis of $\left[\mathrm{CdGe}(\text { phen })_{2}\left(\mu_{2}-\mathrm{OH}\right)_{2}\left(\mathrm{C}_{2} \mathrm{O}_{4}\right)_{2}\right](5)$. The synthetic procedure used to isolate $\left[\mathrm{CdGe}(\text { phen })_{2}\left(\mu_{2}-\mathrm{OH}\right)_{2}\left(\mathrm{C}_{2} \mathrm{O}_{4}\right)_{2}\right]$ as a colorless single-crystalline phase is identical to that described for 1a but using instead $\mathrm{CdC}_{4} \mathrm{H}_{6} \mathrm{O}_{4} \cdot 2 \mathrm{H}_{2} \mathrm{O}$ as the $\mathrm{Cd}^{2+}$ source. The reaction mixture contained $0.100 \mathrm{~g}$ of $\mathrm{GeO}_{2}, 0.360 \mathrm{~g}$ of $\mathrm{H}_{2} \mathrm{C}_{2} \mathrm{O}_{4}$. $\mathrm{H}_{2} \mathrm{O}, 0.080 \mathrm{~g}$ of $\mathrm{CdC}_{4} \mathrm{H}_{6} \mathrm{O}_{4} \cdot 2 \mathrm{H}_{2} \mathrm{O}, 0.200 \mathrm{~g}$ of phen, and $15 \mathrm{~g}$ of $\mathrm{H}_{2} \mathrm{O}$ (approximate molar composition of $1 \mathrm{GeO}_{2}: 3 \mathrm{H}_{2} \mathrm{C}_{2} \mathrm{O}_{4}: 1 /$ $3 \mathrm{CdC}_{4} \mathrm{H}_{6} \mathrm{O}_{4} \cdot 2 \mathrm{H}_{2} \mathrm{O}: 1$ phen). Yield $88.2 \%$ based on $\mathrm{CdC}_{4} \mathrm{H}_{6} \mathrm{O}_{4}$.

Calculated elemental composition (for $\mathrm{C}_{28} \mathrm{H}_{18} \mathrm{~N}_{4} \mathrm{O}_{10} \mathrm{CdGe}$, MW 755.45, \%): C 44.52, N 7.42, H 2.40. Found (\%): C 43.78, N 7.29, $\mathrm{H}$ 2.71. TGA data (weight losses inside parentheses): $30-$ $260{ }^{\circ} \mathrm{C}(-0.6 \%) ; 260-343{ }^{\circ} \mathrm{C}(-36.4 \%) ; 343-598{ }^{\circ} \mathrm{C}(-29.1 \%)$. Selected FT-IR and Raman (inside parentheses and in italics) data (in $\left.\mathrm{cm}^{-1}\right): v(\mathrm{O}-\mathrm{H}$ as bridging ligand $)=3321 \mathrm{~m} ; v_{\text {asym }}(\mathrm{C}-\mathrm{H}$, aromatic rings $)=3074 \mathrm{w},(3080,3052) ; v($ uncoordinated carbonyl groups $)=1723 \mathrm{~s} ; \nu_{\text {asym }}\left(-\mathrm{CO}_{2}^{-}\right)=1680 \mathrm{~s}, 1621 \mathrm{~m}, 1597 \mathrm{~m},(1606)$; $v(\mathrm{C}-\mathrm{C}$, heteroaromatic amines $)=1517 \mathrm{~m},(1452) ; \delta(\mathrm{C}-\mathrm{H})=$ $1428 \mathrm{~s},(1412) ; v_{\mathrm{sym}}\left(-\mathrm{CO}_{2}^{-}\right)=1428 \mathrm{~s}, 1359 \mathrm{~s},(1412,1375,1345$, $1305) ; v(\mathrm{C}-\mathrm{N}$, heteroaromatic amines $)=1359 \mathrm{~s} ;(1345,1305)$; $v(\mathrm{C}-\mathrm{O})=1246 \mathrm{~m}, 1225 \mathrm{~m}, 1138 \mathrm{w},(1257,1196,1156) ; v(\mathrm{C}-\mathrm{C}$ skeletal stretching mode $)=1100 \mathrm{w},(1109) ; v(\mathrm{C}-\mathrm{H})=1053 \mathrm{w}$, $(1055) ; v(\mathrm{C}-\mathrm{C}$ and $\mathrm{Ge}-\mathrm{O})=894 \mathrm{w}, 853 \mathrm{~s}, 808 \mathrm{~m},(865) ; \delta\left(\mathrm{CO}_{2}{ }^{-}\right)$ $=773 \mathrm{w}, 726 \mathrm{~s},(728,711) ; \gamma(\mathrm{C}-\mathrm{H})=642 \mathrm{~m}, 597 \mathrm{~m},(583,556)$; $v(\mathrm{M}-\mathrm{N})$ and $\rho\left(\mathrm{CO}_{2}^{-}\right)=476 \mathrm{~m}, 420 \mathrm{w},(422)$.

Synthesis of $\left[\mathrm{CuGe}(\text { phen })_{2}\left(\mu_{2}-\mathrm{OH}\right)_{2}\left(\mathrm{C}_{2} \mathrm{O}_{4}\right)_{2}\right](6)$. [CuGe(phen $)_{2}-$ $\left.\left(\mu_{2}-\mathrm{OH}\right)_{2}\left(\mathrm{C}_{2} \mathrm{O}_{4}\right)_{2}\right]$ was initially isolated from the reaction vials of 1a as a minor impurity. The pure phase was isolated using the same synthetic procedure with a reaction mixture composed of $0.050 \mathrm{~g}$ of $\mathrm{GeO}_{2}, 0.120 \mathrm{~g}$ of $\mathrm{H}_{2} \mathrm{C}_{2} \mathrm{O}_{4} \cdot \mathrm{H}_{2} \mathrm{O}, 0.100 \mathrm{~g}$ of $\mathrm{CuC}_{4} \mathrm{H}_{6} \mathrm{O}_{4} \cdot \mathrm{H}_{2} \mathrm{O}$, $0.200 \mathrm{~g}$ of phen, and $15 \mathrm{~g}$ of $\mathrm{H}_{2} \mathrm{O}$ (approximate molar composition of $1 \mathrm{GeO}_{2}: 2 \mathrm{H}_{2} \mathrm{C}_{2} \mathrm{O}_{4}: 1 \mathrm{CuC}_{4} \mathrm{H}_{6} \mathrm{O}_{4}: 2$ phen). Yield $70.8 \%$ based on $\mathrm{CuC}_{4} \mathrm{H}_{6} \mathrm{O}_{4} \cdot \mathrm{H}_{2} \mathrm{O}$.

Calculated elemental composition (for $\mathrm{C}_{28} \mathrm{H}_{18} \mathrm{~N}_{4} \mathrm{O}_{10} \mathrm{CuGe}$, MW 706.59; \%): C 47.60, N 7.93, H 2.57. Found (\%): C 47.96, $\mathrm{N} 7.90, \mathrm{H}$ 2.55. TGA data (weight losses inside parentheses): $105-197{ }^{\circ} \mathrm{C}(-0.5 \%) ; 197-342{ }^{\circ} \mathrm{C}(-31.6 \%) ; 342-599{ }^{\circ} \mathrm{C}$ $(-42.4 \%)$. Selected FT-IR and Raman (inside parentheses and in italics) data (in $\left.\mathrm{cm}^{-1}\right): v(\mathrm{O}-\mathrm{H}$ as bridging ligand) $=3396 \mathrm{~m}$; $v_{\text {asym }}(\mathrm{C}-\mathrm{H}$, aromatic rings $)=3086 \mathrm{w},(3078) ; v$ (uncoordinated carbonyl groups $)=1719 \mathrm{~s} ; v_{\text {asym }}\left(-\mathrm{CO}_{2}^{-}\right)=1660 \mathrm{~s}, 1597 \mathrm{~s},(1607)$; $v(\mathrm{C}-\mathrm{C}$, heteroaromatic amines $)=1519 \mathrm{~m},(1457) ; \delta(\mathrm{C}-\mathrm{H})=$ $1428 \mathrm{~s},(1429) ; v_{\mathrm{sym}}\left(-\mathrm{CO}_{2}^{-}\right)=1428 \mathrm{~s}, 1363 \mathrm{~s},(1429,1310)$; $v(\mathrm{C}-\mathrm{N}$, heteroaromatic amines $)=1363 \mathrm{~s} ;(1310) ; v(\mathrm{C}-\mathrm{O})=$ $1296 \mathrm{~m}, 1248 \mathrm{~m}, 1145 \mathrm{w} ; \nu(\mathrm{C}-\mathrm{C}$ skeletal stretching mode $)=1107 \mathrm{w}$, $(1050) ; v(\mathrm{C}-\mathrm{H})=1034 \mathrm{w} ; v(\mathrm{C}-\mathrm{C}$ and $\mathrm{Ge}-\mathrm{O})=854 \mathrm{~m}, 808 \mathrm{~m}$;
$\delta\left(\mathrm{CO}_{2}^{-}\right)=724 \mathrm{~s},(736) ; \gamma(\mathrm{C}-\mathrm{H})=647 \mathrm{w}, 574 \mathrm{w},(560) ; v(\mathrm{M}-\mathrm{N})$ and $\rho\left(\mathrm{CO}_{2}{ }^{-}\right.$out-of-plane $)=483 \mathrm{~m}, 429 \mathrm{w},(432)$.

Single-Crystal X-ray Diffraction. Crystals of compounds $\left[\mathrm{M}(\text { phen })_{3}\right]\left[\mathrm{Ge}\left(\mathrm{C}_{2} \mathrm{O}_{4}\right)_{3}\right] \cdot x \mathrm{H}_{2} \mathrm{O}$ [where $\mathrm{M}^{2+}=\mathrm{Cu}^{2+}(\mathbf{1 a}$ and $\mathbf{1 b}), \mathrm{Fe}^{2+}$ (2a and $\mathbf{2 b}), \mathrm{Ni}^{2+}(\mathbf{3}) ; x=0.2$ for $\left.\mathbf{2 b}\right]$ and $\left[\mathrm{MGe}(\text { phen })_{2}\left(\mu_{2}-\mathrm{OH}\right)_{2^{-}}\right.$ $\left(\mathrm{C}_{2} \mathrm{O}_{4}\right)_{2}$ ] [where $\mathrm{M}^{2+}=\mathrm{Cd}^{2+}(\mathbf{5})$ and $\mathrm{Cu}^{2+}(\mathbf{6})$ ] suitable for singlecrystal X-ray diffraction analysis were manually harvested from the reaction vials and mounted on glass fibers using FOMBLIN Y perfluoropolyether vacuum oil (LVAC 25/6) purchased from Aldrich. ${ }^{45}$ Data were collected in Nonius-based Kappa Bruker diffractrometers equipped with charge-coupled device (CCD) area detectors and $\mathrm{Mo} \mathrm{K} \alpha(\lambda=0.7107 \AA)$ or $\mathrm{Cu} \mathrm{K} \alpha(\lambda=1.54180 \AA)$ (for 3 ) radiation. Data were corrected for Lorenztian and polarization effects. Absorption corrections were applied using the multiscan semiempirical method implemented in SADABS. ${ }^{46}$ Structures were solved using the direct methods of SHELXS-97, ${ }^{47}$ which allowed immediate location of the heaviest atoms. The remaining non-hydrogen atoms were located from difference Fourier maps calculated from successive full-matrix least-squares refinement cycles on $F^{2}$ using SHELXL-97.48 All non-hydrogen atoms were successfully refined using anisotropic displacement parameters.

Hydrogen atoms bound to carbon were located at their idealized positions by employing the HFIX 43 instruction in SHELXL-97 ${ }^{48}$ and included in subsequent refinement cycles in riding motion approximation with isotropic thermal displacement parameters $\left(U_{\text {iso }}\right)$ fixed at $1.2 U_{\mathrm{eq}}$ of the carbon atom to which they were attached. In compounds 5 and $\mathbf{6}$ the hydrogen atoms associated with the $\mu_{2^{-}}$ bridging hydroxyl groups were markedly visible in the last difference Fourier maps synthesis. These atoms have been included in the final structural models with the $\mathrm{O}-\mathrm{H}$ distances restrained to 0.95(1) $\AA$ in order to ensure a chemically reasonable geometry for these moieties, and with $U_{\text {iso }}$ fixed at $1.5 U_{\text {eq }}$ of the parent oxygen atom.

In compound $\mathbf{2 b}$ a partially occupied water molecule of crystallization $[\mathrm{O}(1 \mathrm{~W})]$ was directly located from difference Fourier maps and refined using an isotropic displacement parameter and a fixed partial site occupancy of $20 \%$ (determined previously by unrestrained refinement of this variable). Even though hydrogen atoms belonging to this chemical moiety could not be directly located from difference Fourier maps and no attempt was made to place these in approximate calculated positions, they have been included in the empirical formula of the compound. As mentioned in the previous section, crystals of $\mathbf{6}$ were obtained as a minor phase in the synthesis of 1a. Crystals systematically showed poor quality associated with diffuse scattering at high angle (therefore, the high $R_{\text {int }}$; see Table 1).

Information concerning the crystallographic data collection and structure refinement are collected in Table 1. Selected bond lengths and angles for compounds $\mathbf{1}$ to $\mathbf{3}$ are summarized in Tables 2 and 3 , respectively, while for compounds $\mathbf{5}$ and $\mathbf{6}$ they are collected in Table 9. Geometrical details on the weak $\mathrm{C}-\mathrm{H} \cdots \mathrm{O}$ hydrogenbonding interactions interconnecting cationic $\left[\mathrm{M}(\mathrm{phen})_{3}\right]^{2+}$ and anionic $\left[\mathrm{Ge}\left(\mathrm{C}_{2} \mathrm{O}_{4}\right)_{3}\right]^{2-}$ moieties in compounds $\mathbf{1 - 3}$ are collected

(45) Kottke, T.; Stalke, D. J. Appl. Crystallogr. 1993, 26, 615-619.

(46) Sheldrick, G. M. SADABS v.2.01, Bruker/Siemens Area Detector Absorption Correction Program; Bruker AXS: Madison, WI, 1998.

(47) Sheldrick, G. M. SHELXS-97, Program for Crystal Structure Solution; University of Göttingen: Göttingen, 1997.

(48) Sheldrick, G. M. SHELXL-97, Program for Crystal Structure Refinement; University of Göttingen: Göttingen, 1997. 
Table 3. Octahedral Angles (deg) for $\left\{\mathrm{MN}_{6}\right\}$ and $\left\{\mathrm{GeO}_{6}\right\}$ Coordination Environments Present in Compounds $\mathbf{1}-\mathbf{3}\left(\mathrm{M}^{2+}=\mathrm{Cu}^{2+}, \mathrm{Fe}^{2+}\right.$, or Ni $\left.{ }^{2+}\right)$

\begin{tabular}{|c|c|c|c|c|c|}
\hline & $\mathbf{1 a}\left(\mathrm{Cu}^{2+}\right)$ & $\mathbf{1 b}\left(\mathrm{Cu}^{2+}\right)$ & $\mathbf{2 a}\left(\mathrm{Fe}^{2+}\right)$ & $\mathbf{2 b}\left(\mathrm{Fe}^{2+}\right)$ & $3\left(\mathrm{Ni}^{2+}\right)$ \\
\hline $\mathrm{N}(1)-\mathrm{M}(1)-\mathrm{N}(2)$ & $81.32(9)$ & $75.98(8)$ & $82.58(6)$ & $83.09(9)$ & $80.14(10)$ \\
\hline $\mathrm{N}(1)-\mathrm{M}(1)-\mathrm{N}(3)$ & $99.39(9)$ & $105.32(8)$ & $96.45(6)$ & $93.25(9)$ & $95.43(10)$ \\
\hline $\mathrm{N}(1)-\mathrm{M}(1)-\mathrm{N}(4)$ & $174.52(9)$ & 173.47(8) & $177.27(6)$ & 174.41(9) & 172.97(10) \\
\hline $\mathrm{N}(1)-\mathrm{M}(1)-\mathrm{N}(5)$ & $94.85(9)$ & $89.81(8)$ & $93.83(6)$ & $97.32(9)$ & $91.81(10)$ \\
\hline $\mathrm{N}(1)-\mathrm{M}(1)-\mathrm{N}(6)$ & $91.42(9)$ & $84.19(8)$ & $89.55(6)$ & $91.98(9)$ & $88.78(10)$ \\
\hline $\mathrm{N}(2)-\mathrm{M}(1)-\mathrm{N}(3)$ & $93.74(9)$ & $92.17(9)$ & $92.65(6)$ & $92.12(9)$ & $90.98(10)$ \\
\hline $\mathrm{N}(2)-\mathrm{M}(1)-\mathrm{N}(4)$ & $95.65(9)$ & 98.11(8) & $94.93(6)$ & 92.93(9) & $94.51(10)$ \\
\hline $\mathrm{N}(2)-\mathrm{M}(1)-\mathrm{N}(5)$ & $171.50(9)$ & $165.61(8)$ & $174.87(6)$ & 177.43(9) & $168.24(10)$ \\
\hline $\mathrm{N}(2)-\mathrm{M}(1)-\mathrm{N}(6)$ & $94.49(9)$ & $94.70(9)$ & $93.38(6)$ & $94.83(9)$ & 91.84(10) \\
\hline $\mathrm{N}(3)-\mathrm{M}(1)-\mathrm{N}(4)$ & $76.18(9)$ & $77.42(8)$ & $82.51(6)$ & $82.94(9)$ & $80.01(10)$ \\
\hline $\mathrm{N}(3)-\mathrm{M}(1)-\mathrm{N}(5)$ & $94.37(9)$ & 93.83(8) & $91.40(6)$ & $90.38(9)$ & $98.36(10)$ \\
\hline $\mathrm{N}(3)-\mathrm{M}(1)-\mathrm{N}(6)$ & $167.28(8)$ & $169.42(9)$ & $172.00(6)$ & $171.75(9)$ & $175.28(10)$ \\
\hline $\mathrm{N}(4)-\mathrm{M}(1)-\mathrm{N}(5)$ & $88.75(9)$ & $95.96(8)$ & $88.72(6)$ & $86.82(9)$ & $94.14(10)$ \\
\hline $\mathrm{N}(4)-\mathrm{M}(1)-\mathrm{N}(6)$ & $93.37(9)$ & $93.63(8)$ & $91.73(6)$ & $92.26(9)$ & $95.99(11)$ \\
\hline $\mathrm{N}(5)-\mathrm{M}(1)-\mathrm{N}(6)$ & $77.95(9)$ & $81.38(9)$ & $82.89(6)$ & $82.63(9)$ & $79.34(10)$ \\
\hline $\mathrm{O}(1)-\mathrm{Ge}(1)-\mathrm{O}(3)$ & $85.83(9)$ & $86.52(8)$ & $85.90(6)$ & $85.64(8)$ & $86.57(10)$ \\
\hline $\mathrm{O}(1)-\mathrm{Ge}(1)-\mathrm{O}(5)$ & $87.10(8)$ & $89.49(8)$ & $87.01(6)$ & $88.12(9)$ & $93.26(10)$ \\
\hline $\mathrm{O}(1)-\mathrm{Ge}(1)-\mathrm{O}(7)$ & $172.12(8)$ & 175.57(8) & $172.11(6)$ & $170.87(9)$ & $177.28(10)$ \\
\hline $\mathrm{O}(1)-\mathrm{Ge}(1)-\mathrm{O}(9)$ & $90.29(8)$ & $88.28(8)$ & $90.09(6)$ & $92.15(9)$ & $88.78(11)$ \\
\hline $\mathrm{O}(1)-\mathrm{Ge}(1)-\mathrm{O}(11)$ & $93.86(9)$ & $95.29(8)$ & $94.15(6)$ & $95.06(9)$ & 93.81(11) \\
\hline $\mathrm{O}(3)-\mathrm{Ge}(1)-\mathrm{O}(5)$ & $93.72(9)$ & $95.83(8)$ & $93.75(6)$ & $93.89(9)$ & $93.64(10)$ \\
\hline $\mathrm{O}(3)-\mathrm{Ge}(1)-\mathrm{O}(7)$ & $90.45(9)$ & $92.43(8)$ & $90.83(6)$ & $88.05(9)$ & $90.86(10)$ \\
\hline $\mathrm{O}(3)-\mathrm{Ge}(1)-\mathrm{O}(9)$ & $173.86(8)$ & $171.66(8)$ & $173.93(6)$ & $173.83(9)$ & $173.54(10)$ \\
\hline $\mathrm{O}(3)-\mathrm{Ge}(1)-\mathrm{O}(11)$ & $88.92(9)$ & $88.16(8)$ & $89.28(6)$ & $88.82(9)$ & $89.92(10)$ \\
\hline $\mathrm{O}(5)-\mathrm{Ge}(1)-\mathrm{O}(7)$ & $86.21(8)$ & $86.33(8)$ & $86.04(6)$ & $85.72(9)$ & $86.04(10)$ \\
\hline $\mathrm{O}(5)-\mathrm{Ge}(1)-\mathrm{O}(9)$ & $90.83(9)$ & $90.64(8)$ & $90.59(6)$ & $91.79(9)$ & $91.09(10)$ \\
\hline $\mathrm{O}(5)-\mathrm{Ge}(1)-\mathrm{O}(11)$ & $177.25(9)$ & $173.95(8)$ & $176.83(6)$ & $175.97(9)$ & $172.26(10)$ \\
\hline $\mathrm{O}(7)-\mathrm{Ge}(1)-\mathrm{O}(9)$ & 93.97(8) & $93.24(8)$ & $93.70(6)$ & $94.77(9)$ & $93.86(10)$ \\
\hline $\mathrm{O}(7)-\mathrm{Ge}(1)-\mathrm{O}(11)$ & $93.01(9)$ & $88.98(8)$ & $92.98(6)$ & $91.40(9)$ & $87.04(10)$ \\
\hline $\mathrm{O}(9)-\mathrm{Ge}(1)-\mathrm{O}(11)$ & $86.59(8)$ & $85.82(8)$ & $86.47(6)$ & $85.63(9)$ & $85.93(10)$ \\
\hline
\end{tabular}

Table 4. Geometrical Parameters for the Possible Weak $\mathrm{C}-\mathrm{H} \cdots \mathrm{O}$ Hydrogen-Bonding Interactions Interconnecting $\left[\mathrm{Ge}\left(\mathrm{C}_{2} \mathrm{O}_{4}\right)_{3}\right]^{2-}$ Anions and $\left[\mathrm{Cu}(\text { phen })_{3}\right]^{2+}$ Cations in the Polymorphic Structure of Compound $1 \mathbf{a}^{a}$

\begin{tabular}{lcc}
\hline $\mathrm{C}-\mathrm{H} \cdots \mathrm{O}$ & $d_{\mathrm{C} \cdots \mathrm{O}}(\AA)$ & $\angle(\mathrm{CHO})(\mathrm{deg})$ \\
\hline $\mathrm{C}(7)-\mathrm{H}(7) \cdots \mathrm{O}(6)^{\mathrm{i}}$ & $3.229(4)$ & 125 \\
$\mathrm{C}(8)-\mathrm{H}(8) \cdots \mathrm{O}(8)^{\mathrm{i}}$ & $3.245(4)$ & 132 \\
$\mathrm{C}(14)-\mathrm{H}(14) \cdots \mathrm{O}(10)^{\mathrm{ii}}$ & $3.192(4)$ & 115 \\
$\mathrm{C}(18)-\mathrm{H}(18) \cdots \mathrm{O}(4)$ & $2.997(3)$ & 109 \\
$\mathrm{C}(20)-\mathrm{H}(20) \cdots \mathrm{O}(12)^{\mathrm{iii}}$ & $3.296(4)$ & 138 \\
$\mathrm{C}(21)-\mathrm{H}(21) \cdots \mathrm{O}(11)^{\mathrm{iii}}$ & $3.502(4)$ & 153 \\
$\mathrm{C}(28)-\mathrm{H}(28) \cdots \mathrm{O}(1)^{\mathrm{iv}}$ & $3.147(3)$ & 126 \\
$\mathrm{C}(29)-\mathrm{H}(29) \cdots \mathrm{O}(2)$ & $3.266(4)$ & 117 \\
$\mathrm{C}(30)-\mathrm{H}(30) \cdots \mathrm{O}(2)$ & $3.163(4)$ & 129 \\
$\mathrm{C}(33)-\mathrm{H}(33) \cdots \mathrm{O}(4)^{\mathrm{i}}$ & $3.375(4)$ & 130 \\
$\mathrm{C}(38)-\mathrm{H}(38) \cdots \mathrm{O}(6)^{\mathrm{v}}$ & $3.125(4)$ & 150 \\
$\mathrm{C}(41)-\mathrm{H}(41) \cdots \mathrm{O}(10)^{\mathrm{vi}}$ & $3.515(4)$ &
\end{tabular}

${ }^{a}$ Symmetry transformations used to generate equivalent atoms: (i) $1 / 2$ $-x,-1 / 2+y, 1.5-z$; (ii) $-1.5+x, 1 / 2-y,-1 / 2+z$; (iii) $-x,-y, 2$ $-z$; (iv) $1-x,-y, 2-z$; (v) $1.5-x,-1 / 2+y, 1.5-z$; (vi) $-1 / 2+x$, $1 / 2-y,-1 / 2+z$

in Tables 4-8. Schematic drawings for all structures have been prepared using Crystal Diamond ${ }^{49}$ and the X-seed software platform. ${ }^{50,51}$

Crystallographic data (excluding structure factors) for the structures reported in this paper have been deposited with the Cambridge Crystallographic Data Centre (CCDC) as supplementary publication numbers: CCDC-627717 to -627721 (compounds 1-3), -628767 and -628768 (compounds $\mathbf{5}$ and $\mathbf{6}$, respectively). Copies of the data can be obtained free of charge on application to CCDC, 12 Union Road, Cambridge CB2 2EZ, U.K. [FAX: (+44) 1223 336033; e-mail: deposit@ccdc.cam.ac.uk].

(49) Brandenburg, K. DIAMOND, Version 3.1d; Crystal Impact GbR: Bonn, Germany, 2006.

(50) Barbour, L. J. J. Supramol. Chem. 2001, 1, 189-191.

(51) Atwood, J. L.; Barbour, L. J. Cryst. Growth Des. 2003, 3, 3-8.
Table 5. Geometrical Parameters for the Possible Weak $\mathrm{C}-\mathrm{H} \cdot \cdots \mathrm{O}$ Hydrogen-Bonding Interactions Interconnecting $\left[\mathrm{Ge}\left(\mathrm{C}_{2} \mathrm{O}_{4}\right)_{3}\right]^{2-}$ Anions and $\left[\mathrm{Cu}(\mathrm{phen})_{3}\right]^{2+}$ Cations in the Polymorphic Structure of Compound $1 \mathbf{b}^{a}$

\begin{tabular}{lcc}
\hline $\mathrm{C}-\mathrm{H} \cdots \mathrm{O}$ & $d_{\mathrm{C} \cdots \mathrm{O}}(\AA)$ & $\angle(\mathrm{CHO})(\mathrm{deg})$ \\
\hline $\mathrm{C}(7)-\mathrm{H}(7) \cdots \mathrm{O}(1)^{\mathrm{i}}$ & $3.339(3)$ & 143 \\
$\mathrm{C}(9)-\mathrm{H}(9) \cdots \mathrm{O}(8)^{\mathrm{ii}}$ & $3.393(3)$ & 161 \\
$\mathrm{C}(14)-\mathrm{H}(14) \cdots \mathrm{O}(9)^{\mathrm{iii}}$ & $3.577(3)$ & 166 \\
$\mathrm{C}(15)-\mathrm{H}(15) \cdots \mathrm{O}(6)^{\mathrm{ii}}$ & $3.081(3)$ & 116 \\
$\mathrm{C}(15)-\mathrm{H}(15) \cdots \mathrm{O}(10)^{\mathrm{iii}}$ & $3.400(4)$ & 131 \\
$\mathrm{C}(18)-\mathrm{H}(18) \cdots \mathrm{O}(7)^{\mathrm{iv}}$ & $3.235(3)$ & 115 \\
$\mathrm{C}(19)-\mathrm{H}(19) \cdots \mathrm{O}(10)^{\mathrm{i}}$ & $3.158(3)$ & 115 \\
$\mathrm{C}(20)-\mathrm{H}(20) \cdots \mathrm{O}(10)^{\mathrm{i}}$ & $3.182(3)$ & 111 \\
$\mathrm{C}(21)-\mathrm{H}(21) \cdots \mathrm{O}(10)^{\mathrm{v}}$ & $3.244(3)$ & 137 \\
$\mathrm{C}(27)-\mathrm{H}(27) \cdots \mathrm{O}(7)^{\mathrm{vi}}$ & $3.362(3)$ & 135 \\
$\mathrm{C}(31)-\mathrm{H}(31) \cdots \mathrm{O}(12)^{\mathrm{vii}}$ & $3.360(4)$ & 130 \\
$\mathrm{C}(40)-\mathrm{H}(40) \cdots \mathrm{O}(2)^{\mathrm{viii}}$ & $3.117(4)$ & 116 \\
$\mathrm{C}(41)-\mathrm{H}(41) \cdots \mathrm{O}(2)^{\mathrm{vi}}$ & $3.269(4)$ & 143 \\
$\mathrm{C}(42)-\mathrm{H}(42) \cdots \mathrm{O}(12)^{\mathrm{ix}}$ & $3.273(3)$ &
\end{tabular}

${ }^{a}$ Symmetry transformations used to generate equivalent atoms: (i) $1-$ $x, 1 / 2+y, 1 / 2-z$; (ii) $1-x, 1-y,-z$; (iii) $-1+x, 1 / 2-y,-1 / 2+$ $z$; (iv) $1-x,-y,-z$; (v) $-1+x, y, z$; (vi) $1-x,-1 / 2+y, 1 / 2-z$; (vii) $1-x, 1 / 2+y, 1 / 2-z$; (viii) $1-x, 1 / 2+y, 1 / 2-z$; (ix) $1-x,-y$, $-z$.

Powder X-ray Diffraction. PXRD data of $\left[\mathrm{Co}(\mathrm{phen})_{3}\right][\mathrm{Ge}-$ $\left.\left(\mathrm{C}_{2} \mathrm{O}_{4}\right)_{3}\right]$ (4) were collected at ambient temperature on a X'Pert MPD Philips diffractometer $(\mathrm{Cu} \mathrm{K} \alpha$ X-radiation, $\lambda=1.54060 \AA$ ) equipped with a $\mathrm{X}^{\prime}$ Celerator detector, a curved graphite-monochromated radiation, and a flat-plate sample holder in a BraggBrentano para-focusing optics configuration $(40 \mathrm{kV}, 50 \mathrm{~mA})$. Intensity data were collected in continuous scanning mode in the range ca. $4 \leq 2 \theta^{\circ} \leq 55$.

The PXRD pattern was indexed with the routines provided with the program DICVOL0 $4^{52}$ using the first 20 well-resolved reflec-

(52) Boultif, A.; Louer, D. J. Appl. Crystallogr. 2004, 37, 724-731. 
Table 6. Geometrical Parameters for the Possible Weak $\mathrm{C}-\mathrm{H} \cdots \mathrm{O}$ Hydrogen-Bonding Interactions Interconnecting $\left[\mathrm{Ge}\left(\mathrm{C}_{2} \mathrm{O}_{4}\right)_{3}\right]^{2-}$ Anions and $\left[\mathrm{Fe}(\mathrm{phen})_{3}\right]^{2+}$ Cations in $\mathbf{2} \mathbf{a}^{a}$

\begin{tabular}{ccc}
\hline $\mathrm{C}-\mathrm{H} \cdots \mathrm{O}$ & $d_{\mathrm{C} \cdots \mathrm{O}}(\AA)$ & $\angle(\mathrm{CHO})(\mathrm{deg})$ \\
\hline $\mathrm{C}(7)-\mathrm{H}(7) \cdots \mathrm{O}(6)^{\mathrm{i}}$ & $3.187(2)$ & 124 \\
$\mathrm{C}(8)-\mathrm{H}(8) \cdots \mathrm{O}(8)^{\mathrm{i}}$ & $3.294(4)$ & 135 \\
$\mathrm{C}(18)-\mathrm{H}(18) \cdots \mathrm{O}(4)$ & $3.028(2)$ & 105 \\
$\mathrm{C}(20)-\mathrm{H}(20) \cdots \mathrm{O}(12)^{\mathrm{ii}}$ & $3.369(3)$ & 128 \\
$\mathrm{C}(21)-\mathrm{H}(21) \cdots \mathrm{O}(11)^{\mathrm{ii}}$ & $3.551(3)$ & 163 \\
$\mathrm{C}(28)-\mathrm{H}(28) \cdots \mathrm{O}(2)^{\mathrm{iii}}$ & $3.146(2)$ & 126 \\
$\mathrm{C}(30)-\mathrm{H}(30) \cdots \mathrm{O}(2)$ & $3.186(2)$ & 108 \\
$\mathrm{C}(33)-\mathrm{H}(33) \cdots \mathrm{O}(4)^{\mathrm{i}}$ & $3.388(3)$ & 137 \\
$\mathrm{C}(38)-\mathrm{H}(38) \cdots \mathrm{O}(6)^{\mathrm{iv}}$ & $3.157(3)$ & 125 \\
$\mathrm{C}(39)-\mathrm{H}(39) \cdots \mathrm{O}(6)^{\mathrm{iv}}$ & $3.246(2)$ & 118 \\
$\mathrm{C}(41)-\mathrm{H}(41) \cdots \mathrm{O}(10)^{\mathrm{v}}$ & $3.564(3)$ & 156
\end{tabular}

${ }^{a}$ Symmetry transformations used to generate equivalent atoms: (i) 1.5 $-x,-1 / 2+y, 1.5-z$; (ii) $2-x,-y, 1-z$; (iii) $1-x,-y, 1-z$; (iv) $1 / 2-x,-1 / 2+y, 1.5-z$; (v) $1 / 2+x, 1 / 2-y, 1 / 2+z$.

Table 7. Geometrical Parameters for the Possible Weak $\mathrm{C}-\mathrm{H} \cdots \mathrm{O}$ Hydrogen-Bonding Interactions Interconnecting $\left[\mathrm{Ge}\left(\mathrm{C}_{2} \mathrm{O}_{4}\right)_{3}\right]^{2-}$ Anions and $\left[\mathrm{Fe}(\mathrm{phen})_{3}\right]^{2+}$ Cations in the Solvate Structure of Compound $\mathbf{2} \mathbf{b}^{a}$

\begin{tabular}{ccc}
\hline $\mathrm{C}-\mathrm{H} \cdots \mathrm{O}$ & $d_{\mathrm{C} \cdots \mathrm{O}}(\AA)$ & $\angle(\mathrm{CHO})(\mathrm{deg})$ \\
\hline $\mathrm{C}(14)-\mathrm{H}(14) \cdots \mathrm{O}(4)^{\mathrm{i}}$ & $3.362(4)$ & 131 \\
$\mathrm{C}(15)-\mathrm{H}(15) \cdots \mathrm{O}(5)^{\mathrm{i}}$ & $3.417(4)$ & 160 \\
$\mathrm{C}(16)-\mathrm{H}(16) \cdots \mathrm{O}(6)^{\mathrm{ii}}$ & $3.513(4)$ & 162 \\
$\mathrm{C}(20)-\mathrm{H}(20) \cdots \mathrm{O}(2)^{\mathrm{iii}}$ & $3.335(5)$ & 133 \\
$\mathrm{C}(30)-\mathrm{H}(30) \cdots \mathrm{O}(7)^{\mathrm{iv}}$ & $3.196(4)$ & 127 \\
$\mathrm{C}(31)-\mathrm{H}(31) \cdots \mathrm{O}(9)^{\mathrm{v}}$ & $3.205(5)$ & 142 \\
$\mathrm{C}(32)-\mathrm{H}(32) \cdots \mathrm{O}(10)^{\mathrm{vi}}$ & $3.225(4)$ & 120 \\
$\mathrm{C}(39)-\mathrm{H}(39) \cdots \mathrm{O}(2)$ & $3.110(3)$ & 122 \\
$\mathrm{C}(40)-\mathrm{H}(40) \cdots \mathrm{O}(4)^{\mathrm{vii}}$ & $3.117(3)$ & 120 \\
$\mathrm{C}(41)-\mathrm{H}(41) \cdots \mathrm{O}(4)^{\mathrm{vii}}$ & $3.181(4)$ & 117 \\
$\mathrm{C}(42)-\mathrm{H}(42) \cdots \mathrm{O}(3)^{\mathrm{iv}}$ & $3.037(3)$ & 102 \\
$\mathrm{C}(42)-\mathrm{H}(42) \cdots \mathrm{O}(4)^{\mathrm{iv}}$ & $3.468(3)$ &
\end{tabular}

${ }^{a}$ Symmetry transformations used to generate equivalent atoms: (i) $2-$ $x, 2-y, z$; (ii) $2+x, 1+y, z$; (iii) $2-x, 2-y, 1-z$; (iv) $1+x, 1+$ $y, z ;$ (v) $1+x, y, z$; (vi) $1-x, 2-y, 1-z$; (vii) $1-x, 2-y, z$.

Table 8. Geometrical Parameters for the Possible Weak $\mathrm{C}-\mathrm{H} \cdots \mathrm{O}$ Hydrogen-Bonding Interactions Interconnecting $\left[\mathrm{Ge}\left(\mathrm{C}_{2} \mathrm{O}_{4}\right)_{3}\right]^{2-}$ Anions and $\left[\mathrm{Ni}(\text { phen })_{3}\right]^{2+}$ Cations in $\mathbf{3}^{a}$

\begin{tabular}{ccc}
\hline $\mathrm{C}-\mathrm{H} \cdots \mathrm{O}$ & $d_{\mathrm{C} \cdots \mathrm{O}}(\AA)$ & $\angle(\mathrm{CHO})(\mathrm{deg})$ \\
\hline $\mathrm{C}(9)-\mathrm{H}(9) \cdots \mathrm{O}(10)^{\mathrm{i}}$ & $3.358(4)$ & 137 \\
$\mathrm{C}(14)-\mathrm{H}(14) \cdots \mathrm{O}(8)^{\mathrm{ii}}$ & $3.129(4)$ & 128 \\
$\mathrm{C}(19)-\mathrm{H}(19) \cdots \mathrm{O}(8)^{\mathrm{i}}$ & $3.211(4)$ & 127 \\
$\mathrm{C}(20)-\mathrm{H}(20) \cdots \mathrm{O}(6)^{\mathrm{i}}$ & $3.295(4)$ & 132 \\
$\mathrm{C}(26)-\mathrm{H}(26) \cdots \mathrm{O}(4)^{\mathrm{iii}}$ & $3.183(5)$ & 115 \\
$\mathrm{C}(30)-\mathrm{H}(30) \cdots \mathrm{O}(10)^{\mathrm{iv}}$ & $3.045(5)$ & 112 \\
$\mathrm{C}(32)-\mathrm{H}(32) \cdots \mathrm{O}(2)^{\mathrm{v}}$ & $3.313(5)$ & 133 \\
$\mathrm{C}(33)-\mathrm{H}(33) \cdots \mathrm{O}(1)^{\mathrm{v}}$ & $3.510(5)$ & 158 \\
$\mathrm{C}(40)-\mathrm{H}(40) \cdots \mathrm{O}(11)^{\mathrm{v}}$ & $3.148(5)$ & 126 \\
$\mathrm{C}(40)-\mathrm{H}(40) \cdots \mathrm{O}(12)^{\mathrm{vi}}$ & $3.650(5)$ & 173 \\
$\mathrm{C}(42)-\mathrm{H}(42) \cdots \mathrm{O}(12)^{\mathrm{iv}}$ & $3.159(5)$ & 131
\end{tabular}

${ }^{a}$ Symmetry transformations used to generate equivalent atoms: (i) 1.5 $-x,-1 / 2+y, 1.5-z$; (ii) $1 / 2-x,-1 / 2+y, 1.5-z$; (iii) $1 / 2-x, 1 / 2$ $-y,-1 / 2-z$; (iv) $-1+x, y,-1+z$; (v) $2-x,-y, 1-z$; (vi) $1-x,-y$, $1-z$

tions (located using the derivative-based peak search algorithm provided with Fullprof. $2 \mathrm{k})^{53,54}$ and a fixed absolute error on each line of $0.03^{\circ} 2 \theta$. Initial unit cell metrics were obtained with reasonable figures-of-merit: $\mathrm{M}(20)^{55}=12.5$ and $\mathrm{F}(20)^{56}=30.2$; zero shift of $-0.0436^{\circ}$. Analysis of the systematic absences using CHECKCELL ${ }^{57}$ unambiguously confirmed space group $P 2_{1} / n$. A

(53) Rodriguez-Carvajal, J. FULLPROF-A Program for Rietveld Refinement and Pattern Matching Analysis, Abstract of the Satellite Meeting on Powder Diffraction of the XV Congress of the IUCR, Toulouse, France, 1990; p 127.
Le Bail whole-powder-diffraction-pattern profile fitting ${ }^{58}$ (see Figure $\mathrm{S} 1$ in the Supporting Information) was performed with the FullProf.2k software package, ${ }^{53,54}$ employing a typical pseudo-Voigt peak-shape function, and in the last stages of the fitting process the unit cell parameters and typical profile parameters, such as scale factor, zero shift, Caglioti function values, and two asymmetry parameters were allowed to refine freely. Fixed background points were employed. The refined unit cell parameters converged to $a=$ 19.204(2) $\AA, b=20.839(2) \AA, c=9.511(1) \AA$, and $\beta=95.066-$ $(6)^{\circ}\left(R_{\text {Bragg }}=1.06 \%\right.$ and $\left.\chi^{2}=3.79\right)$

\section{Results and Discussion}

A series of highly crystalline heterodimetallic complexes composed of tris(oxalato- $O, O^{\prime}$ )germanate anions and cationic complexes of transition metals (M) coordinated to $1,10^{\prime}$ phenanthroline (phen) residues have been isolated using mild hydrothermal synthesis, from reaction mixtures containing germanium(IV) oxide, oxalic acid, phen, and various $\mathrm{M}$ salts (see Experimental Section for details). Compounds have been formulated as $\left[\mathrm{M}(\mathrm{phen})_{3}\right]\left[\mathrm{Ge}\left(\mathrm{C}_{2} \mathrm{O}_{4}\right)_{3}\right] \cdot x \mathrm{H}_{2} \mathrm{O}$ [where $\mathrm{M}^{2+}=$ $\mathrm{Cu}^{2+}(\mathbf{1} \mathbf{a}$ and $\mathbf{1 b}), \mathrm{Fe}^{2+}$ (2a and 2b), $\mathrm{Ni}^{2+}(\mathbf{3})$, and $\mathrm{Co}^{2+}(\mathbf{4})$; $x=0.2$ for compound $\mathbf{2 b}$ ] on the basis of single-crystal or powder X-ray diffraction studies and elemental analysis. EDS studies provided information on (1) the presence of Ge and the metallic centers on individual crystals of each compound and (2) the ratios of Ge:M, typically 1:1.

Crystalline phases were directly isolated from the autoclave contents in generous yields and usually as large single crystals, except for $\mathbf{4}$ which could only be synthesized as a microcrystalline powder (Figure 1). Phase purity and homogeneity of the bulk samples of $\mathbf{1 b}$ and $\mathbf{2 - 4}$ have been confirmed by comparing the experimental powder X-ray diffraction patterns with simulations based on single-crystal data. Compound 1a was systematically isolated with a small amount of compound $\mathbf{6}$ which could not be eliminated either at the synthesis stage or after. Nevertheless, according to the CHN elemental composition of several representative bulk samples of 1a we determined that the amount of this impurity was quite small. However, during our synthetic attempts to eliminate this second-phase (by varying the composition of the reaction mixture used to isolate 1a) a second polymorphic (and pure) phase was isolated for a slightly higher amount of phen in the reaction mixture (see Experimental Section for details on the synthetic procedures).

It is also of considerable interest to mention that for $\mathrm{Fe}^{2+}$ two structures could also be isolated, $\left[\mathrm{Fe}(\mathrm{phen})_{3}\right]\left[\mathrm{Ge}\left(\mathrm{C}_{2} \mathrm{O}_{4}\right)_{3}\right]$ (2a) and $\left[\mathrm{Fe}(\text { phen })_{3}\right]\left[\mathrm{Ge}\left(\mathrm{C}_{2} \mathrm{O}_{4}\right)_{3}\right] \cdot 0.2 \mathrm{H}_{2} \mathrm{O}(\mathbf{2 b})$, with the latter

(54) Roisnel, T.; Rodriguez-Carvajal, J. WinPLOTR [June 2005]-A Windows Tool for Powder Diffraction Pattern Analysis. Materials Science Forum, Proceedings of the Seventh European Powder Diffraction Conference (EPDIC 7); Delhez, R., Mittenmeijer, E. J., Eds.; 2000, pp 118-123.

(55) Boultif, A.; Louer, D. J. Appl. Crystallogr. 1991, 24, 987-993.

(56) Louer, D. In Automatic Indexing: Procedures and Applications, Accuracy in Powder Diffraction II; Gaithersburg, MD, 1992; pp 92104

(57) Laugier, J.; Bochu, B. CHECKCELL-A Software Performing Automatic Cell/Space Group Determination, Collaborative Computational Project Number 14 (CCP14), Laboratoire des Matériaux et du Génie Physique de l'Ecole Supérieure de Physique de Grenoble (INPG), France, 2000

(58) LeBail, A.; Duroy, H.; Fourquet, J. L. Mater. Res. Bull. 1988, 23, $447-452$. 

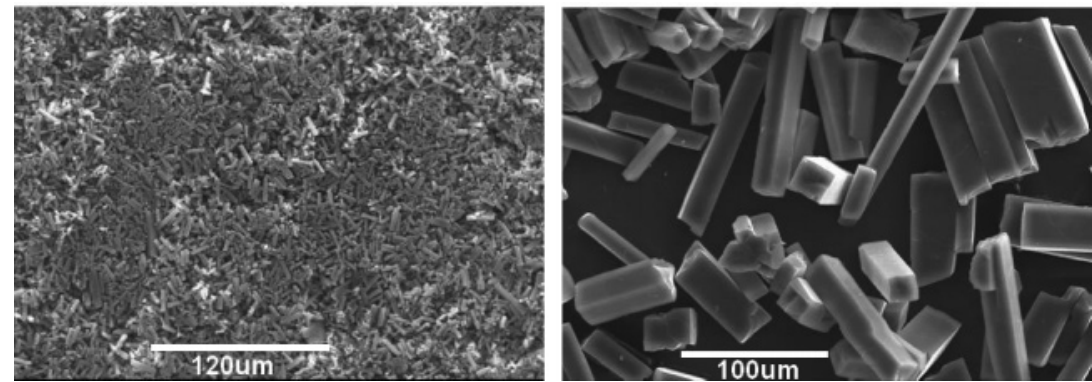

Figure 1. SEM images of $\left[\mathrm{M}(\text { phen })_{3}\right]\left[\mathrm{Ge}\left(\mathrm{C}_{2} \mathrm{O}_{4}\right)_{3}\right]$ crystals with $\mathrm{M}^{2+}=\mathrm{Ni}^{2+}$ (3) (right) and $\mathrm{Co}^{2+}$ (4) (left).

Table 9. Selected Bond Lengths $(\AA)$ and Angles (deg) for the Octahedral $\left\{\mathrm{MN}_{4} \mathrm{O}_{2}\right\}$ and $\left\{\mathrm{GeO}_{6}\right\}$ Coordination Environments Present in Compounds 5 and $6\left(\mathrm{M}^{2+}=\mathrm{Cd}^{2+} \text { or } \mathrm{Cu}^{2+}\right)^{a}$

\begin{tabular}{|c|c|c|c|c|c|}
\hline & \multicolumn{2}{|c|}{ bond lengths $(\AA)$} & & \multicolumn{2}{|c|}{ bond angles (deg) } \\
\hline & $5\left(\mathrm{Cd}^{2+}\right)$ & $6\left(\mathrm{Cu}^{2+}\right)$ & & $5\left(\mathrm{Cd}^{2+}\right)$ & $6\left(\mathrm{Cu}^{2+}\right)$ \\
\hline $\mathrm{M}(1)-\mathrm{N}(1)$ & $2.311(1)$ & $1.989(1)$ & $\mathrm{N}(1)-\mathrm{M}(1)-\mathrm{N}(2)$ & $72.32(4)$ & $81.4(4)$ \\
\hline $\mathrm{M}(1)-\mathrm{N}(2)$ & $2.339(1)$ & $2.138(1)$ & $\mathrm{N}(1)-\mathrm{M}(1)-\mathrm{O}(5)$ & 88.63(4) & $89.2(4)$ \\
\hline \multirow{7}{*}{$\mathrm{M}(1)-\mathrm{O}(5)$} & $2.297(1)$ & $2.206(9)$ & $\mathrm{N}(1)-\mathrm{M}(1)-\mathrm{N}(1)^{\mathrm{i}}$ & $157.40(6)$ & $170.2(6)$ \\
\hline & & & $\mathrm{N}(1)-\mathrm{M}(1)-\mathrm{N}(2)^{\mathrm{i}}$ & 93.03(4) & $93.2(4)$ \\
\hline & & & $\mathrm{N}(1)-\mathrm{M}(1)-\mathrm{O}(5)^{\mathrm{i}}$ & $110.67(4)$ & $98.9(4)$ \\
\hline & & & $\mathrm{N}(2)-\mathrm{M}(1)-\mathrm{O}(5)$ & $97.08(4)$ & $89.3(3)$ \\
\hline & & & $\mathrm{N}(2)-\mathrm{M}(1)-\mathrm{N}(2)^{\mathrm{i}}$ & $100.39(6)$ & $112.7(5)$ \\
\hline & & & $\mathrm{N}(2)-\mathrm{M}(1)-\mathrm{O}(5)^{\mathrm{i}}$ & $162.11(4)$ & $158.0(3)$ \\
\hline & & & $\mathrm{O}(5)-\mathrm{M}(1)-\mathrm{O}(5)^{\mathrm{i}}$ & $65.81(5)$ & $68.8(4)$ \\
\hline $\mathrm{Ge}(1)-\mathrm{O}(1)$ & $1.941(1)$ & $1.951(8)$ & $\mathrm{O}(1)-\mathrm{Ge}(1)-\mathrm{O}(3)$ & $83.89(4)$ & $83.8(4)$ \\
\hline $\mathrm{Ge}(1)-\mathrm{O}(3)$ & $1.899(1)$ & $1.898(8)$ & $\mathrm{O}(1)-\mathrm{Ge}(1)-\mathrm{O}(5)$ & $92.18(5)$ & $175.7(4)$ \\
\hline \multirow[t]{7}{*}{$\mathrm{Ge}(1)-\mathrm{O}(5)$} & $1.817(1)$ & $1.823(8)$ & $\mathrm{O}(1)-\mathrm{Ge}(1)-\mathrm{O}(1)^{\mathrm{i}}$ & $89.19(6)$ & $89.2(5)$ \\
\hline & & & $\mathrm{O}(1)-\mathrm{Ge}(1)-\mathrm{O}(3)^{\mathrm{i}}$ & 87.14(4) & $87.5(4)$ \\
\hline & & & $\mathrm{O}(1)-\mathrm{Ge}(1)-\mathrm{O}(5)^{\mathrm{i}}$ & 175.64(4) & $92.4(4)$ \\
\hline & & & $\mathrm{O}(3)-\mathrm{Ge}(1)-\mathrm{O}(5)$ & $97.12(5)$ & $92.2(4)$ \\
\hline & & & $\mathrm{O}(3)-\mathrm{Ge}(1)-\mathrm{O}(3)^{\mathrm{i}}$ & $167.40(6)$ & $167.8(5)$ \\
\hline & & & $\mathrm{O}(3)-\mathrm{Ge}(1)-\mathrm{O}(5)^{\mathrm{i}}$ & $92.04(5)$ & $96.7(4)$ \\
\hline & & & $\mathrm{O}(5)-\mathrm{Ge}(1)-\mathrm{O}(5)^{\mathrm{i}}$ & $86.75(7)$ & $86.3(6)$ \\
\hline
\end{tabular}

${ }^{a}$ Symmetry transformation used to generate equivalent atoms: (i) $-x, y, 1.5-z$.

being a solvate of the former and typically obtained for a slightly higher concentration of oxalic acid in the reaction mixture (see Experimental Section). This extra partially occupied water molecule of crystallization induces long-range structural modifications in the crystal packing, namely, a reduction in crystal symmetry from the monoclinic to the triclinic crystal systems (see structural details in the following paragraphs).

Structural Details of the $\left[\mathrm{Ge}\left(\mathrm{C}_{2} \mathrm{O}_{4}\right)_{3}\right]^{2-}$ Anion. The chemical moiety common to structures $\mathbf{1 - 4}$ is the divalent tris(oxalate- $\left.O, O^{\prime}\right)$ germanate anion, $\left[\mathrm{Ge}\left(\mathrm{C}_{2} \mathrm{O}_{4}\right)_{3}\right]^{2-}$ (Figure 2 and Scheme 1). A search in the literature and in the $\mathrm{CSD}^{39,40}$ reveals only a handful of crystallographic reports ${ }^{41-44}$ describing this anion which, invariably, shows identical coordination geometry for all compounds, including those reported herein. The $\mathrm{Ge}^{4+}$ center appears coordinated to three oxalate anions (coordinated via a typical anti,anti-chelate bidentate fashion), describing a slightly distorted $\left\{\mathrm{GeO}_{6}\right\}$ octahedral coordination fashion as depicted in Figure 2. The $\mathrm{Ge}-\mathrm{O}$ bonds (for all five crystal structure determinations reported here) are found in the $1.865(2)-1.897(2) \AA$ (Table 2 ), while the cis and trans $\mathrm{O}-\mathrm{Ge}-\mathrm{O}$ octahedral angles are instead in the 85.64(8)-95.83(8) ${ }^{\circ}$ and 170.87(9)-177.28$(10)^{\circ}$ ranges, respectively (Table 3 ). These values are in good agreement with those described for the aforementioned related compounds available in the literature, which show an average $\mathrm{Ge}-\mathrm{O}$ bond length of ca. $1.88 \AA$ plus average cis (or chelate bite angle) and trans $\mathrm{O}-\mathrm{Ge}-\mathrm{O}$ octahedral angles of $85.7^{\circ}$ and $173.6^{\circ}$, respectively. ${ }^{41-44}$

Crystal Structure Description of $\left[\mathrm{Cu}(\text { phen })_{3}\right][\mathrm{Ge}-$ $\left.\left(\mathrm{C}_{2} \mathrm{O}_{4}\right)_{3}\right]$ (1a and 1b). Two crystalline forms of [Cu(phen $\left.)_{3}\right]-$ $\left[\mathrm{Ge}\left(\mathrm{C}_{2} \mathrm{O}_{4}\right)_{3}\right]$, $\mathbf{1 a}$ and $\mathbf{1 b}$, have been isolated from hydrothermal synthesis (see Experimental Section), crystallizing in the $P 2_{1} / n$ and $P 2_{1} / c$ monoclinic space groups, respectively. For both crystalline materials the asymmetric unit comprises two discrete (and charged) crystallographically independent divalent residues: one $\left[\mathrm{Cu}(\text { phen })_{3}\right]^{2+}$ cation plus one $\left[\mathrm{Ge}\left(\mathrm{C}_{2} \mathrm{O}_{4}\right)_{3}\right]^{2-}$ anion (Figure 3).

The crystallographically independent $\mathrm{Cu}^{2+}$ centers in 1a and $\mathbf{1 b}$ are coordinated by three phen organic ligands via a typical $N, N$-chelating coordination fashion, leading to distorted $\left\{\mathrm{CuN}_{6}\right\}$ octahedral coordination geometries evidencing the typical Jahn-Teller distortion expected for these $\mathrm{d}^{9}$ metallic centers: while the equatorial $\mathrm{Cu}-\mathrm{N}$ bond lengths are found in the $2.021(2)-2.059(2) \AA$ range, the apical interactions are much longer and within the 2.275(2)2.374(2) ^ range (Table 2), thus leading to a tetragonal distortion of the $\left\{\mathrm{CuN}_{6}\right\}$ octahedra. The former bond lengths are well within the expected values, as revealed by a search in the CSD for structures containing $\left[\mathrm{Cu}(\text { phen })_{3}\right]^{2+}$ cations (15 entries; range $2.01-2.34 \AA$ ), however, the latter values are slightly longer, in particular for $\mathbf{1 b}$ (see Table 2). Such abnormal long $\mathrm{Cu}-\mathrm{N}$ interactions with phen residues can be rationalized by taking into account intermolecular interac- 


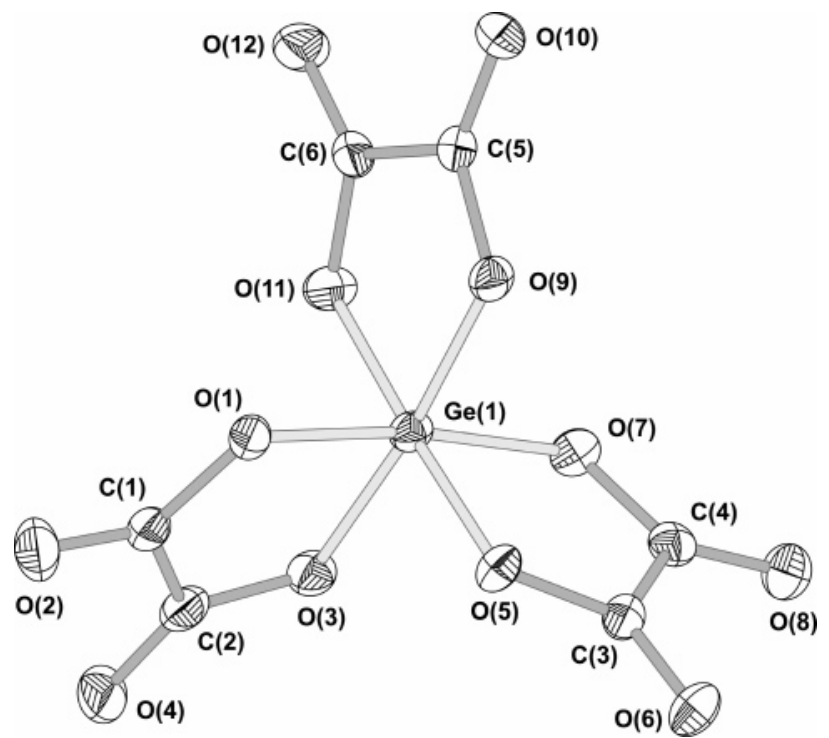

Figure 2. Anionic $\left[\mathrm{Ge}\left(\mathrm{C}_{2} \mathrm{O}_{4}\right)_{3}\right]^{2-}$ fragment common to structures 1-4. The represented structure was taken from the structure of complex $\mathbf{1 b}$ and is represented with thermal ellipsoids drawn at the $50 \%$ probability level and showing the labeling scheme for all atoms. For selected bond lengths and angles related to this moiety in structures $\mathbf{1}-\mathbf{3}$ see Tables 2 and 3, respectively.

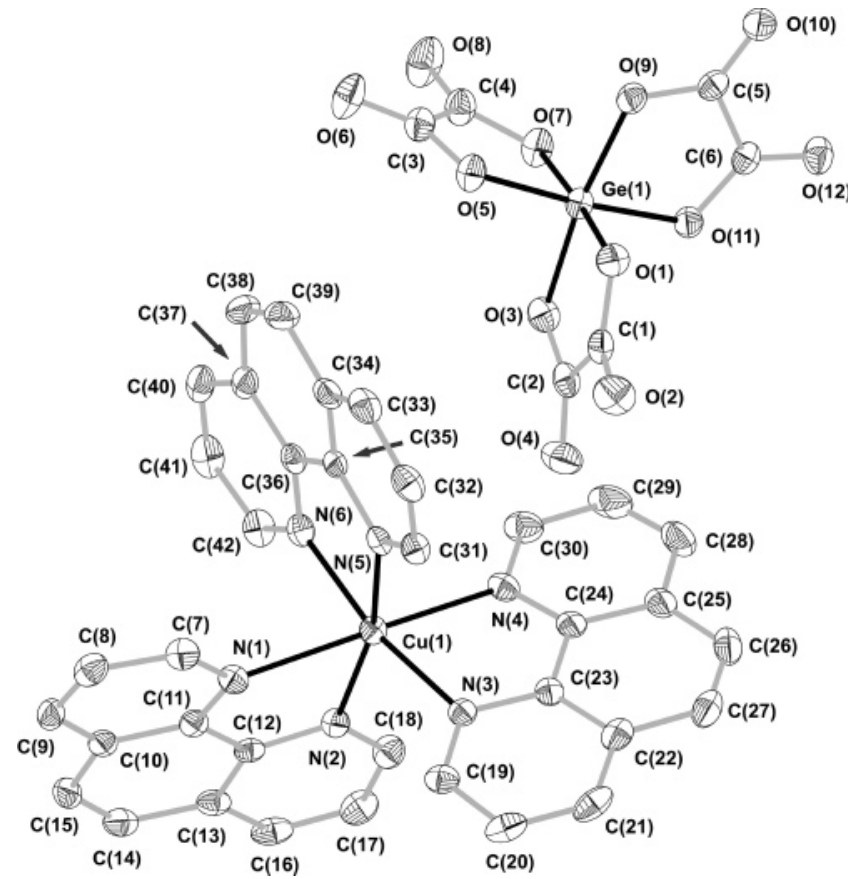

Figure 3. Two crystallographic independent chemical moieties, $\left[\mathrm{M}(\mathrm{phen})_{3}\right]^{2+}$ and $\left[\mathrm{Ge}\left(\mathrm{C}_{2} \mathrm{O}_{4}\right)_{3}\right]^{2-}$, composing the crystal structures of $\mathbf{1 - 3}$ from the refined structure of polymorph $\left.\left[\mathrm{Cu}(\mathrm{phen})_{3}\right]\left[\mathrm{Ge}\left(\mathrm{C}_{2} \mathrm{O}_{4}\right)_{3}\right](\mathbf{1 b})\right\}$ and showing the labeling scheme for all non-hydrogen atoms. Atoms are drawn as thermal ellipsoids at the $50 \%$ probability level, and hydrogen atoms have been omitted for clarity. For selected bond lengths and angles see Tables 2 and 3 , respectively.

tions, in particular the weak $\mathrm{C}-\mathrm{H} \cdots \mathrm{O}$ interactions which lead to local distortions in order to promote a more effective close packing (see following paragraphs). Structural distortions associated with $\mathbf{1 b}$ are particularly well demonstrated by the cis octahedral angles whose range is the largest of all related structures reported herein (see Table 3).

The most remarkable structural feature which differentiates 1a and 1b structures concerns the number and type of intermolecular $\mathrm{C}-\mathrm{H} \cdots \mathrm{O}$ weak hydrogen bonds (not shown; Tables 4 and 5 collect some geometrical details of the most relevant and chemically feasible interactions) plus interactions of the $\mathrm{C}-\mathrm{H} \cdots \pi$ type and $\pi-\pi$ stacking between neighboring phen residues belonging to distinct cationic residues (Figure 4)..$^{59}$ These supramolecular networks of weak intermolecular interactions establish physical connections between individual moieties and are responsible for completely distinct packing arrangements, as shown in Figure 5. Moreover, these interactions are ultimately the main reason responsible for the isolation of two distinct crystalline forms. In $\mathbf{1 a}$ the $\left[\mathrm{Cu}(\text { phen })_{3}\right]^{2+}$ cationic residues pack in chain-like arrangements along the [100] and [001] crystallographic directions (Figure $4 \mathrm{a}$ and $4 \mathrm{~b}$ ). While down the [100] direction adjacent $\left[\mathrm{Cu}(\text { phen })_{3}\right]^{2+}$ cations interact only via $\mathrm{C}-\mathrm{H} \cdots \pi$ contacts [see Figure $4 \mathrm{a} ; \pi$ represents the centroid of the neighboring adjacent aromatic ring; approximate $\mathrm{C} \cdots \pi$ distances: $H(19) 3.34 \AA$, $H(20) 3.22 \AA, H(40) 3.31 \AA, H(41)$ $3.36 \AA$ ] , along the [001] direction connections between neighboring complexes alternate between $\pi-\pi$ stacking and $\mathrm{C}-\mathrm{H} \cdot \cdots \pi$ interactions $[\mathrm{C}(8)-\mathrm{H}(8) \cdots \pi$ with $\mathrm{C} \cdots \pi$ distance of $3.22 \AA$ A see Figure 4b]. In $\mathbf{1 b}$ only one structurally relevant $\mathrm{C}-\mathrm{H} \cdots \pi$ interaction was observed along the [001] direction of the unit cell [Figure $4 \mathrm{c} ; \mathrm{C}(29)-\mathrm{H}(29) \cdots \pi$ with $\mathrm{C} \cdots \pi$ average distance of $3.20 \AA$ ]. Noteworthy is the fact that this contact seems to promote the unusually long $\mathrm{Cu}-\mathrm{N}$ bonds discussed above. Indeed, in order to maximize the geometry associated with this $\mathrm{C}-\mathrm{H} \cdots \pi$ interaction, one coordinated phen residue needs to be slightly rotated. This leads, on one hand, to longer bond lengths (because of the structural rigidity of this organic ligand) and, on the other, to the large cis octahedral angles (see above). It is also of considerable importance to mention that adjacent phen residues in $\mathbf{1 b}$ are too far apart (average distance of ca. $4.3 \AA$ ), thus invalidating the occurrence of $\pi-\pi$ stacking (as also depicted in Figure $4 c)$.

Crystal Structure Description of $\left[\mathrm{Fe}(\text { phen })_{3}\right][\mathrm{Ge}-$ $\left.\left(\mathrm{C}_{2} \mathrm{O}_{4}\right)_{3}\right]$ (2a) and $\left[\mathrm{Fe}(\text { phen })_{3}\right]\left[\mathrm{Ge}\left(\mathrm{C}_{2} \mathrm{O}_{4}\right)_{3}\right] \cdot 0.2 \mathrm{H}_{2} \mathrm{O}$ (2b). When $\mathrm{Fe}^{2+}$ is included in the reaction mixtures (see Experimental Section) two pseudo-polymorphic crystalline forms could be isolated with empirical formulas [ $\left.\mathrm{Fe}(\mathrm{phen})_{3}\right]$ $\left[\mathrm{Ge}\left(\mathrm{C}_{2} \mathrm{O}_{4}\right)_{3}\right]$ and $\left[\mathrm{Fe}(\text { phen })_{3}\right]\left[\mathrm{Ge}\left(\mathrm{C}_{2} \mathrm{O}_{4}\right)_{3}\right] \cdot 0.2 \mathrm{H}_{2} \mathrm{O}$ for compounds $\mathbf{2 a}$ and $\mathbf{2 b}$, respectively. Pseudo-polymorphism is defined as crystalline forms of a given compound (host) that differ in the chemical nature or stoichiometry of the included solvent molecules (guest) and refers to crystalline forms with solvent molecules as structurally relevant features of the structure (isolated lattice sites, lattice channels, or metalion-coordinated solvates). ${ }^{60,61}$

The striking difference between these two compounds is the presence of one partially occupied (1/5) water molecule of crystallization in the asymmetric unit of $\mathbf{2} \mathbf{b}$, a unique feature among the series of compounds reported here. It is

(59) Russell, V.; Scudder, M.; Dance, I. Dalton Trans. 2001, 789-799. (60) Robin, A. Y.; Fromm, K. M. Coord. Chem. Rev. 2006, 250, $2127-$ 2157.

(61) Bernstein, J. Organic Solid State Chemistry. In Studies in Organic Chemistry; Desiraju, G. R., Ed.; Elsevier: Amsterdam, 1987; Vol. 32 . 


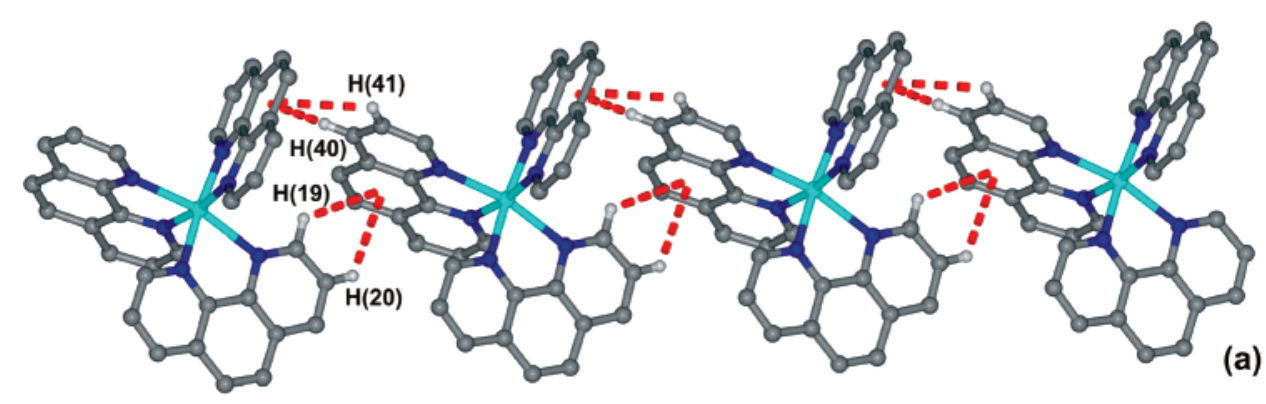

(a)
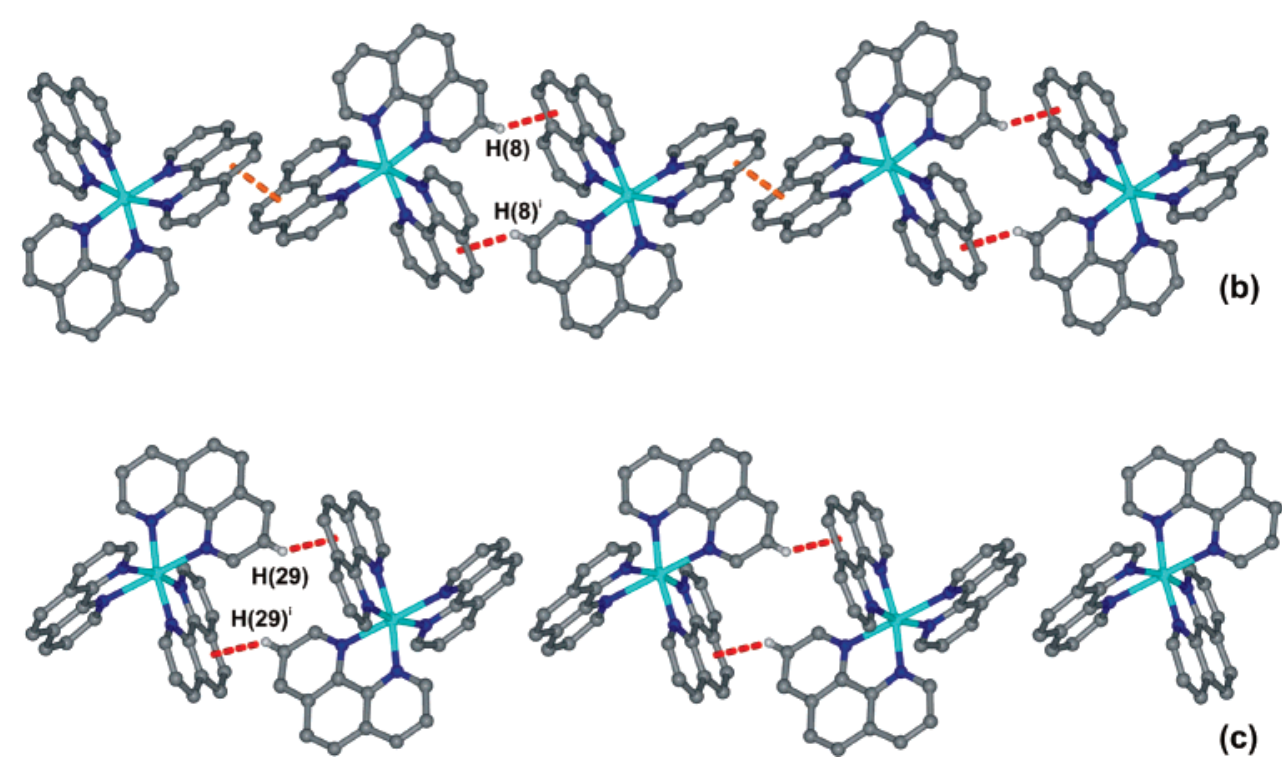

Figure 4. Close packing of cationic $\left[\mathrm{Cu}(\mathrm{phen})_{3}\right]^{2+}$ fragments along the (a) [100] and (b) [001] crystallographic directions of polymorph 1a and along the (c) [001] direction for polymorph $\mathbf{1 b}$, emphasizing the $\mathrm{C}-\mathrm{H} \cdots \pi$ and $\pi-\pi$ interactions (dashed lines) interconnecting these moieties. For clarity, only the hydrogen atoms involved in the represented interactions are shown. Geometrical details (approximate $\mathrm{C} \cdots \pi$ distances) on structurally relevant $\mathrm{C}-\mathrm{H} \cdots \pi$ interactions $(\pi$ represents the centroid of the aromatic ring): $\mathrm{C}(19)-\mathrm{H}(19) \cdots \pi 3.34 \AA$; $\mathrm{C}(20)-\mathrm{H}(20) \cdots \pi 3.22 \AA, \mathrm{C}(40)-\mathrm{H}(40) \cdots \pi 3.31 \AA$, $\mathrm{C}(41)-\mathrm{H}(41) \cdots \pi 3.36 \AA$; $\mathrm{C}(8)-\mathrm{H}(8) \cdots \pi 3.22 \AA$; $\mathrm{C}(29)-\mathrm{H}(29) \cdots \pi 3.20 \AA$.

also of considerable interest to note that this occurrence seems to be the promoting structural reason for the decrease of crystal symmetry: $\mathbf{2} \mathbf{b}$ crystallizes in the triclinic $P \overline{1}$ space group, while 2a (as for all remaining heterometallic complex structures) is monoclinic (in this case described in the $P 2_{1} / n$ space group) or higher. Disregarding the presence of water, the asymmetric units of $\mathbf{2 a}$ and $\mathbf{2} \mathbf{b}$ share many similarities with those described for the two previous compounds, comprising two crystallographically independent and charged ions: $\left[\mathrm{Fe}(\text { phen })_{3}\right]^{2+}$ and $\left[\mathrm{Ge}\left(\mathrm{C}_{2} \mathrm{O}_{4}\right)_{3}\right]^{2-}$. The $\mathrm{Fe}^{2+}$ centers exhibit an almost regular $\left\{\mathrm{FeN}_{6}\right\}$ octahedral coordination environment with the $\mathrm{Fe}-\mathrm{O}$ bond lengths and cis- and trans$\mathrm{N}-\mathrm{Fe}-\mathrm{N}$ octahedral angles being found in the 1.972(2)2.002(2) $\AA$ (Table 2) and 82.58(8)-97.32(9) ${ }^{\circ}$ and 171.75(9) $-177.43(10)^{\circ}$ (Table 3) ranges, respectively. These values are in agreement with those found in similar structures containing $\left[\mathrm{Fe}(\text { phen })_{3}\right]^{2+}$ complexes, as revealed by a search in the CSD and in the literature.

The partially occupied water molecule of crystallization $[\mathrm{O}(1 \mathrm{~W})]$ is strongly hydrogen bonded to two neighboring divalent $\left[\mathrm{Ge}\left(\mathrm{C}_{2} \mathrm{O}_{4}\right)_{3}\right]^{2-}$ anionic fragments, establishing a connection between these two moieties (Figure 6): $\mathrm{O}(1 \mathrm{~W}$ ) donates its two hydrogen atoms to $\mathrm{O}(7)$ and $\mathrm{O}(10)$ from distinct anions, with $d_{0} \cdots \mathrm{O}$ of $3.031(2)$ and 2.881(1) $\AA$, respectively. As also depicted in Figure 6, these bonding interactions occur in symmetry-related pairs with the two water molecules being separated by $4.004(2) \AA$. The motif can be described by the $R_{4}{ }^{4}(16)$ graph set notation. ${ }^{62}$

As for 1a and $\mathbf{1 b}$ (see previous subsection), the crystal structure of these two pseudo-polymorphic compounds is assembled by extensive networks of weak $\mathrm{C}-\mathrm{H} \cdots \mathrm{O}$ interactions involving the charged species. Chemically (and structurally) possible $\mathrm{C} \cdots \mathrm{O}$ intermolecular distances span from 3.028(2) to 3.564(3) $\AA$ for 2a (Table 6) and from 3.037(3) to 3.513(4) $\AA$ for $\mathbf{2 b}$ (Table 7). In the same way as for $\mathbf{1 a}$, in 2a intermolecular interactions are composed of both $\mathrm{C}-$ $\mathrm{H} \cdots \pi$ and $\pi-\pi$ contacts between phen molecules belonging to neighboring $\left[\mathrm{Fe}(\mathrm{phen})_{3}\right]^{2+}$ complexes. While along the [100] direction connections are assured by only $\mathrm{C}-\mathrm{H} \cdots \pi$ interactions (as in Figure 4a), parallel to the [001] direction an alternation between $\mathrm{C}-\mathrm{H} \cdots \pi$ and $\pi-\pi$ contacts is registered (as in Figure $4 b$ ). ${ }^{59}$ In $\mathbf{2 b}$ only $\mathrm{C}-\mathrm{H} \cdots \pi$ interactions (running parallel to the [100] crystallographic direction) are structurally relevant as physical connections between adjacent cationic residues (Figure 7).

The presence of the extra solvent molecule in $\mathbf{2 b}$ also induces a completely distinct packing arrangement. While

(62) Bernstein, J.; Davis, R. E.; Shimoni, L.; Chang, N. L. Angew. Chem., Int. Ed. Engl. 1995, 34, 1555-1573. 
Shi et al.

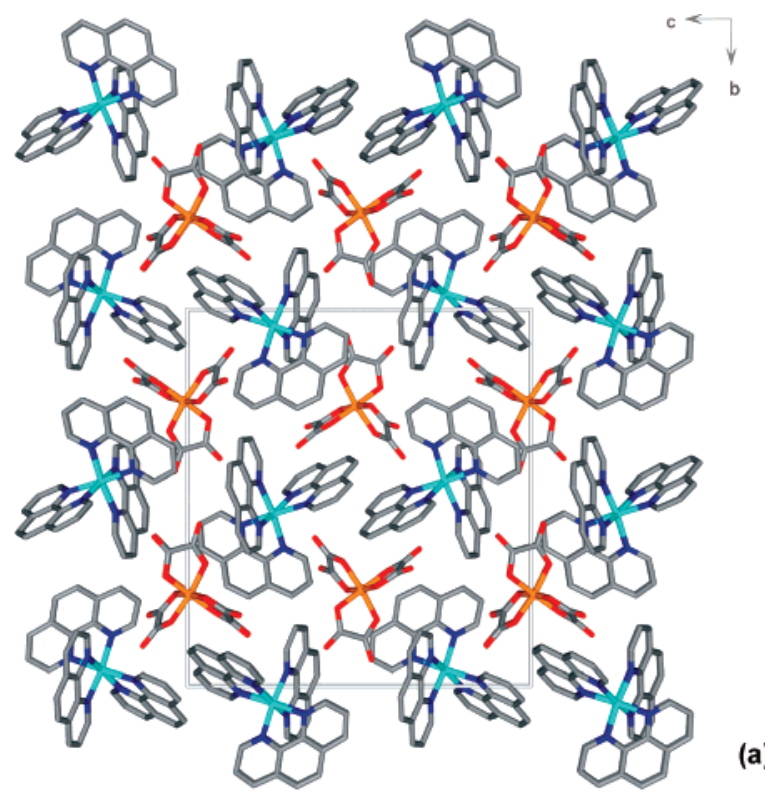

(a)

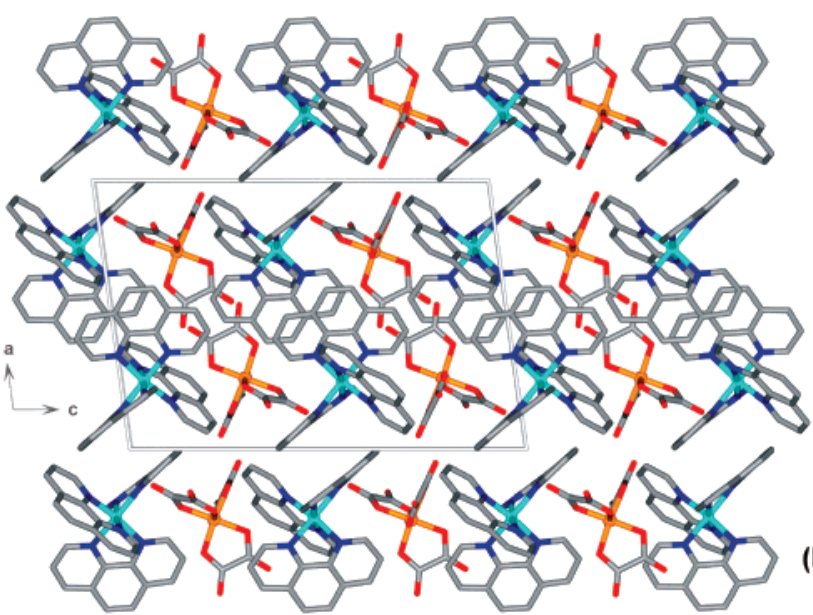

Figure 5. Schematic representations of the crystal packing of (a) 1a (along the [100] direction) and (b) $\mathbf{1 b}$ (along the [010] direction). Hydrogen atoms have been omitted for clarity.

for 2a the crystal packing is identical to that previously described for $\mathbf{1 a}$ (as in Figure 5a), in $\mathbf{2 b}\left[\mathrm{Fe}(\text { phen })_{3}\right]^{2+}$ cations alternate along the [010] direction of the unit cell with $\left[\mathrm{Ge}\left(\mathrm{C}_{2} \mathrm{O}_{4}\right)_{3}\right]^{2-}$ anions, almost perfectly aligned in a typical eclipsed fashion (Figure 8). Interstitial spaces are occupied by the hydrogen-bonded water molecules of crystallization.

Crystallographic Aspects of $\left[\mathrm{Ni}(\text { phen })_{3}\right]\left[\mathrm{Ge}\left(\mathrm{C}_{2} \mathrm{O}_{4}\right)_{3}\right](3)$ and $\left[\mathrm{Co}(\text { phen })_{3}\right]\left[\mathrm{Ge}\left(\mathrm{C}_{2} \mathrm{O}_{4}\right)_{3}\right](\mathbf{4})$. $\left[\mathrm{Ni}(\text { phen })_{3}\right]\left[\mathrm{Ge}\left(\mathrm{C}_{2} \mathrm{O}_{4}\right)_{3}\right](\mathbf{3})$ crystallizes in the monoclinic $P 2_{1} / n$ space group, as revealed by single-crystal X-ray diffraction (Table 1), and its molecular structure shares striking resemblances with those previously described for compounds $\mathbf{1 a}$ and $\mathbf{2 a}$ (see previous sections for details on the crystal structure). Relevant geometrical details associated with the $\mathrm{Ge}^{4+}$ and $\mathrm{Ni}^{2+}$ coordination environments of $\mathbf{3}$ are collected in Tables 2 and 3 .

The compound with $\mathrm{Co}^{2+}$, formulated as $\left[\mathrm{Co}(\mathrm{phen})_{3}\right][\mathrm{Ge}-$ $\left.\left(\mathrm{C}_{2} \mathrm{O}_{4}\right)_{3}\right]$ (4) on the basis of elemental analysis (and other supporting techniques), was also found to be isostructural

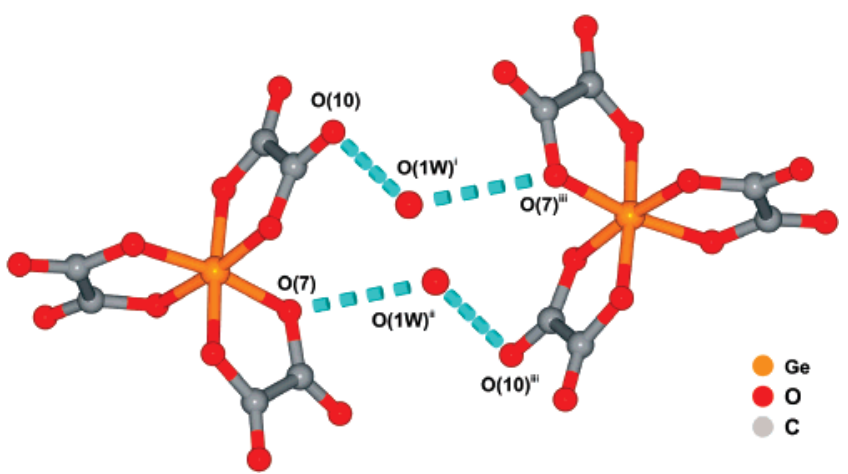

Figure 6. $\mathrm{O}-\mathrm{H} \cdots \mathrm{O}$ hydrogen-bonding interactions (dashed blue lines) involving the partially occupied water molecule of crystallization in pseudopolymorph $\mathbf{2 b}$, [ $\left.\mathrm{Fe}(\text { phen })_{3}\right]\left[\mathrm{Ge}\left(\mathrm{C}_{2} \mathrm{O}_{4}\right)_{3}\right] \cdot 0.2 \mathrm{H}_{2} \mathrm{O}$, interconnecting neighboring $\left[\mathrm{Ge}\left(\mathrm{C}_{2} \mathrm{O}_{4}\right)_{3}\right]^{2-}$ anionic fragments and leading to formation of a $R_{4}^{4}(16)$ graph set motif. Geometrical aspects of the represented hydrogen bonds: $\mathrm{O}(1 \mathrm{~W})^{\mathrm{i}} \cdots \mathrm{O}(7)^{\mathrm{iii}}$ with $d_{\mathrm{O}} \cdots \mathrm{O}$ of $3.031(2) \AA$ 的 $\mathrm{O}(1 \mathrm{~W})^{\mathrm{i}} \cdots \mathrm{O}(10)$ with $d_{\mathrm{O}} \cdots \mathrm{O}$ of 2.881(1) A. Symmetry transformations used to generate equivalent atoms: (i) $1-x, 2-y, 1-z$; (ii) $-1+x, y, z$; (iii) $-x, 2-y, 1-z$.

with 1a and 2a from powder X-ray diffraction studies (see details in the Experimental Section).

Crystal Structure Description of [MGe(phen $)_{2}\left(\mu_{2^{-}}-\mathrm{OH}\right)_{2^{-}}$ $\left.\left(\mathrm{C}_{2} \mathrm{O}_{4}\right)_{2}\right]$ (where $\mathrm{M}^{2+}=\mathrm{Cd}^{2+}$ and $\mathrm{Cu}^{2+}$ for 5 and 6). The neutral hetero-binuclear compounds formulated as $[\mathrm{MGe}-$ (phen $)_{2}\left(\mu_{2}-\mathrm{OH}\right)_{2}\left(\mathrm{C}_{2} \mathrm{O}_{4}\right)_{2}$ ] $\left[\right.$ where $\mathrm{M}^{2+}=\mathrm{Cd}^{2+}(\mathbf{5})$ and $\mathrm{Cu}^{2+}$ (6) ] represent, to the best of our knowledge, the first examples of binuclear complexes containing a neutral bis(oxalate- $\left.O, O^{\prime}\right)$ germanium fragment, $\left[\mathrm{Ge}\left(\mathrm{C}_{2} \mathrm{O}_{4}\right)_{2}\right]$, connected by two $\mu_{2}$-bridging hydroxyl groups to a cationic $\left[\mathrm{M}(\mathrm{phen})_{2}\right]^{2+}$ fragment (Figure 9). Moreover, a search in the literature reveals that these two isostructural complexes are also the first examples of hydroxyl-bridged $\left[\mathrm{M}(\mathrm{phen})_{2}\right]^{2+}$ fragments, and only a handful of structures are known to contain $\mu_{2-}$ oxo bridges involving this fragment. ${ }^{63-75}$

The neutral bis(oxalate- $O, O^{\prime}$ ) germanium fragment, [Ge$\left.\left(\mathrm{C}_{2} \mathrm{O}_{4}\right)_{2}\right]$, shares structural similarities with the $\left[\mathrm{Ge}\left(\mathrm{C}_{2} \mathrm{O}_{4}\right)_{3}\right]^{2-}$ complex discussed above and present in structures 1-4. However, in $\mathbf{5}$ and $\mathbf{6}$ the $\mathrm{Ge}^{4+}$ center is only coordinated to

(63) Xiao, D. R.; Xu, Y.; Hou, Y.; Wang, E. B.; Wang, S. T.; Li, Y. G.; $\mathrm{Xu}, \mathrm{L}$; Hu, C. W. Eur. J. Inorg. Chem. 2004, 1385-1388.

(64) Qi, Y. J.; Wang, Y. H.; Li, H. M.; Cao, M. H.; Hu, C. W.; Wang, E. B.; Hu, N. H.; Jia, H. Q. J. Mol. Struct. 2003, 650, 123-129.

(65) Devi, R. N.; Burkholder, E.; Zubieta, J. Inorg. Chim. Acta 2003, 348, $150-156$.

(66) Liu, C. M.; Zhang, D. Q.; Xiong, M.; Dai, M. Q.; Hu, H. M.; Zhu, D. B. J. Coord. Chem. 2002, 55, 1327-1335.

(67) Lu, Y.; Wang, E. B.; Yuan, M.; Li, Y. G.; Xu, L.; Hu, C. W.; Hu, N. H.; Jia, H. Q. Solid State Sci. 2002, 4, 449-453.

(68) Lu, Y.; Wang, E. B.; Yuan, M.; Li, Y. G.; Hu, C. W.; Hu, N. H.; Jia, H. Q. J. Mol. Struct. 2002, 607, 189-194.

(69) Liu, C. M.; Hou, Y. L.; Zhang, J.; Gao, S. Inorg. Chem. 2002, 41 140 .

(70) Zhang, X. M.; Tong, M. L.; Chen, X. M. Chem. Commun. 2000 $1817-1818$

(71) Mizutani, M.; Tomosue, S.; Kinoshita, H.; Jitsukawa, K.; Masuda, H.; Einaga, H. Bull. Chem. Soc. Jpn. 1999, 72, 981-988.

(72) Xu, J. Q.; Wang, R. Z.; Yang, G. Y.; Xing, Y. H.; Li, D. M.; Bu, W M.; Ye, L.; Fan, Y. G.; Yang, G. D.; Xing, Y.; Lin, Y. H.; Jia, H. Q. Chem. Commun. 1999, 983-984.

(73) Reddy, K. R.; Rajasekharan, M. V.; Arulsamy, N.; Hodgson, D. J. Inorg. Chem. 1996, 35, 2283-2286.

(74) Vincent, J. M.; Menage, S.; Latour, J. M.; Bousseksou, A.; Tuchagues, J. P.; Decian, A.; Fontecave, M. Angew. Chem., Int. Ed. Engl. 1995 34, 205-207.

(75) Tokii, T.; Ide, K.; Nakashima, M.; Koikawa, M. Chem. Lett. 1994, $441-444$

6512 Inorganic Chemistry, Vol. 46, No. 16, 2007 


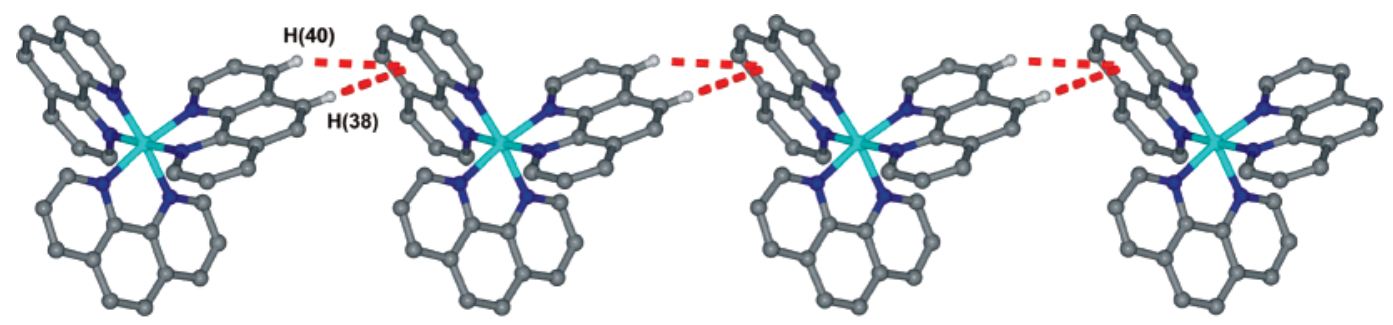

Figure 7. Close packing of cationic $\left[\mathrm{Cu}(\text { phen })_{3}\right]^{2+}$ fragments in $\mathbf{2 b}$ along the [100] crystallographic direction, emphasizing the $\mathrm{C}-\mathrm{H} \cdots \pi$ interactions between neighboring residues. For clarity, only the hydrogen atoms involved in the represented interactions are shown. Geometrical details (approximate $\mathrm{C} \cdots \pi$ distances) on structurally relevant $\mathrm{C}-\mathrm{H} \cdots \pi$ interactions $(\pi$ represents the centroid of the aromatic ring): $\mathrm{C}(38)-\mathrm{H}(38) \cdots \pi 3.39 \AA$; $\mathrm{C}(40)-\mathrm{H}(40) \cdots \pi$ $3.66 \AA$.

two $O, O$-chelate oxalate anions, with the two remaining vacant positions in the coordination sphere being occupied by two $\mu_{2}$-bridging hydroxyl groups (Figure 9). The coordination environment of $\mathrm{Ge}^{4+}$ centers can thus be envisaged as distorted octahedra, $\left\{\mathrm{GeO}_{6}\right\}$, with the $\mathrm{Ge}-\mathrm{O}$ bonds being found (for the two complexes) between 1.8166(10) and 1.951(8) $\AA$ while the cis and trans $\mathrm{O}-\mathrm{Ge}-\mathrm{O}$ angles are in the $83.8(4)-97.12(5)^{\circ}$ and $167.40(6)-175.7(4)^{\circ}$ ranges, respectively (Table 9). The presence of $\mu_{2}$-bridging hydroxyl groups induces in the $\mathrm{Ge}^{4+}$ centers octahedral distortions greater than those observed for the $\left[\mathrm{Ge}\left(\mathrm{C}_{2} \mathrm{O}_{4}\right)_{3}\right]^{2-}$ anions (Table 3 ) with this being essentially attributed to the stronger bonding nature of these chemical moieties. Consequently, the registered $\mathrm{Ge}-\mathrm{OH}$ bond distances are statistically smaller (below ca. $1.82 \AA$ ) than those with oxalate anions (always greater than ca. $1.90 \AA$; Table 9), even though they compare well with the only report in the literature containing a $\mathrm{Ge}-$ $(\mathrm{OH})-\mathrm{M}$ bridge $(d(\mathrm{Ge}-\mathrm{OH})$ of about $1.78 \AA)$ and present in an open-framework containing germanium clusters. ${ }^{76}$ Moreover, the trans effect of these $\mu_{2}-\mathrm{OH}$ groups in the $\left\{\mathrm{GeO}_{6}\right\}$ octahedra is also markedly present with the opposite $\mathrm{Ge}-\mathrm{O}_{\text {oxalate }}$ bonds [1.9413(10) and 1.951(8) $\AA$ for $\mathbf{5}$ and $\mathbf{6}$, respectively] being the longest among all the structures reported here.

The $\mathrm{M}$ centers exhibit distorted octahedral coordination geometries composed by two symmetry-related $N, N$-chelating phen ligands plus two symmetry-related $\mu_{2}$-bridging hydroxyl groups, $\left\{\mathrm{MN}_{4} \mathrm{O}_{2}\right\}$ (Figure 9): the $\mathrm{M}-(\mathrm{N}, \mathrm{O})$ bond lengths are found in the 2.2967(10)-2.3385(12) and 1.989(10)2.206(9) $\AA$ ranges for 5 and 6, respectively; cis and trans internal octahedral angles can be found in the 65.81(5)$100.39(6)$ and $157.40(6)-162.11(4)^{\circ}$ (for 5) and in the 68.8$(4)-112.7(5)^{\circ}$ and $158.0(3)-170.2(6)^{\circ}$ ranges, respectively (see Table 9 for details). Noteworthy is the fact that for $\mathbf{6}$ the $\left\{\mathrm{CuN}_{4} \mathrm{O}_{2}\right\}$ coordination environment is not as significantly distorted as those registered for $\mathbf{1 a}$ and $\mathbf{1 b}$, and consequently the Jahn-Teller distortion is not so markedly visible. It is feasible to assume that this smaller tetragonal distortion for $\mathbf{6}$ seems to arise mainly due to the presence of three crystallographically independent $\mathrm{Cu}-(\mathrm{N}, \mathrm{O})$ bond lengths, which are all statistically distinct as summarized in Table 9 . The intermetallic $\mathrm{Ge}(1) \cdots \mathrm{M}(1)$ separations across the hydroxyl $\mu_{2}$ bridge are 3.2487(3) and 3.1510(27) $\AA$ for 5 (Cd) and $\mathbf{6}(\mathrm{Cu})$, respectively.

(76) Lin, Z. E.; Zhang, J.; Zheng, S. T.; Yang, G. Y. Microporous Mesoporous Mater. 2004, 74, 205-211.

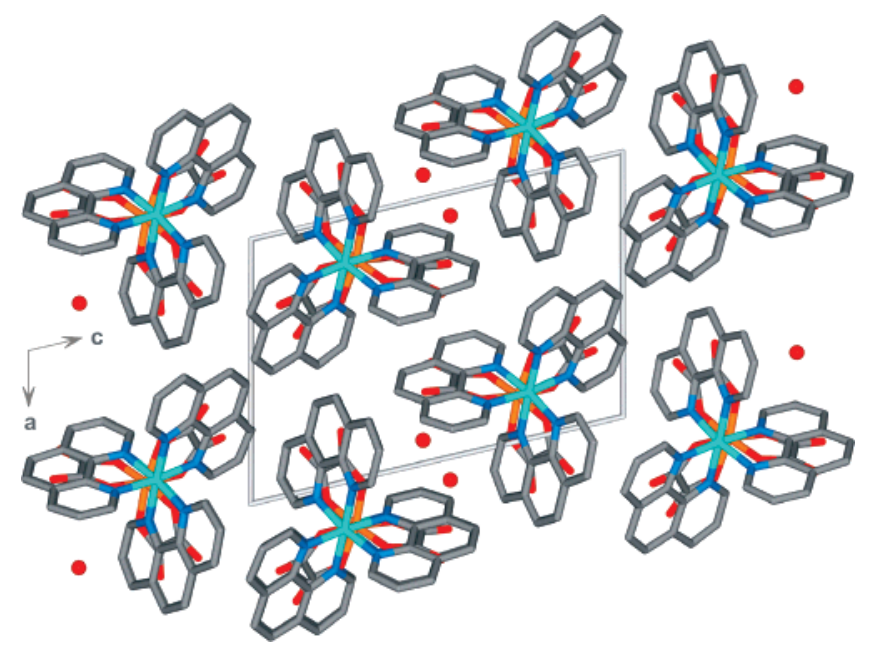

Figure 8. Ball-and-stick packing arrangement of $\mathbf{2 b}$ viewed along the crystallographic [010] direction. Hydrogen atoms have been omitted for clarity.

The close packing in the solid state of individual [MGe(phen $\left.)_{2}\left(\mu_{2}-\mathrm{OH}\right)_{2}\left(\mathrm{C}_{2} \mathrm{O}_{4}\right)_{2}\right]$ complexes is mediated by a number of intermolecular interactions, in particular strong and highly directional $\mathrm{O}-\mathrm{H} \cdots \mathrm{O}$ hydrogen bonds involving anionic [Ge$\left.\left(\mu_{2}-\mathrm{OH}\right)_{2}\left(\mathrm{C}_{2} \mathrm{O}_{4}\right)_{2}\right]^{2-}$ fragments belonging to neighboring complexes: $\mu_{2}$-bridging hydroxyl groups donate their hydrogen atoms to neighboring oxalate anions $[\mathrm{O}(5)-$ $\mathrm{H}(5 \mathrm{~A}) \cdots \mathrm{O}(2)^{\mathrm{i}}$; for $\mathbf{5}, d(\mathrm{O} \cdots \mathrm{O})=2.7699(15) \AA$ with $\angle(\mathrm{OHO})$ $=170.3(2)^{\circ}$; for $\mathbf{6}, d(\mathrm{O} \cdots \mathrm{O})=2.7791(13) \AA$ with $\angle(\mathrm{OHO})$ $=168.0(1)^{\circ}$; symmetry operation (i) $\left.-x,-y, 1-z\right]$, leading to formation of a $R_{2}^{2}(12)$ graph set motif. ${ }^{62}$ The reciprocity of these hydrogen-bonding interactions leads to formation of $1 \mathrm{D}$ supramolecular tapes, ${ }_{\infty}^{2}\left[\mathrm{MGe}(\mathrm{phen})_{2}\left(\mu_{2}-\mathrm{OH}\right)_{2}\left(\mathrm{C}_{2} \mathrm{O}_{4}\right)_{2}\right]$, as depicted in Figure 10a. Interactions between adjacent tapes are assured by cooperative $\pi-\pi$ stacking involving the phen residues coordinated to the $\mathrm{M}$ centers (Figure 11).

Thermal Analysis. The thermal stability (under a continuous air flow) of $\left[\mathrm{M}(\text { phen })_{3}\right]\left[\mathrm{Ge}\left(\mathrm{C}_{2} \mathrm{O}_{4}\right)_{3}\right] \cdot x \mathrm{H}_{2} \mathrm{O}$ [with $\mathrm{M}^{2+}=$ $\mathrm{Cu}^{2+}(\mathbf{1 a}$ and $\mathbf{1 b}), \mathrm{Fe}^{2+}(\mathbf{2 a}$ and $\mathbf{2 b}), \mathrm{Ni}^{2+}(\mathbf{3})$, and $\left.\mathrm{Co}^{2+}(\mathbf{4})\right]$ and $\left[\mathrm{MGe}(\text { phen })_{2}\left(\mu_{2}-\mathrm{OH}\right)_{2}\left(\mathrm{C}_{2} \mathrm{O}_{4}\right)_{2}\right]\left[\right.$ where $\mathrm{M}^{2+}=\mathrm{Cd}^{2+}(\mathbf{5})$ and $\left.\mathrm{Cu}^{2+}(\mathbf{6})\right]$ was investigated between ambient temperature and ca. $600{ }^{\circ} \mathrm{C}$ with the registered thermograms (see Figure S2) clearly evidencing a similar behavior for all compounds belonging to a given family.

This study was particularly informative regarding the subtle composition difference between pseudo-polymorphs $\left[\mathrm{Fe}(\text { phen })_{3}\right]\left[\mathrm{Ge}\left(\mathrm{C}_{2} \mathrm{O}_{4}\right)_{3}\right]$ (2a) and $\left[\mathrm{Fe}(\text { phen })_{3}\right]\left[\mathrm{Ge}\left(\mathrm{C}_{2} \mathrm{O}_{4}\right)_{3}\right] \cdot$ $0.2 \mathrm{H}_{2} \mathrm{O}(\mathbf{2 b})$. Indeed, for the latter compound between 
Shi et al.

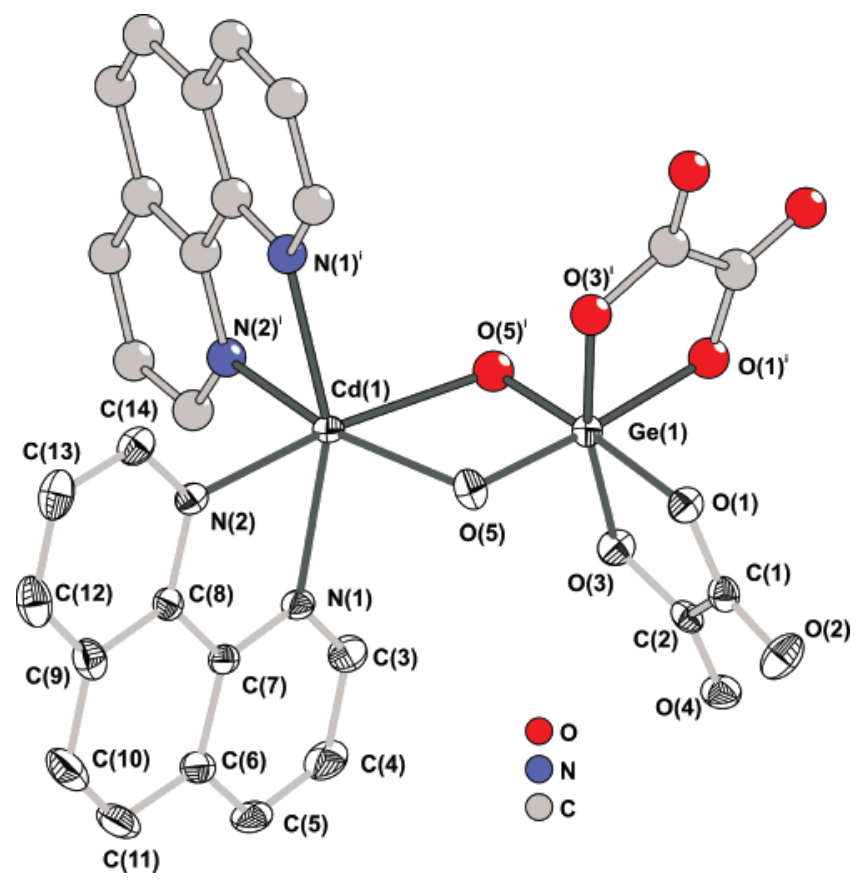

Figure 9. Schematic representation of the binuclear $\left[\mathrm{CdGe}(\mathrm{phen})_{2}\left(\mu_{2^{-}}\right.\right.$ $\mathrm{OH})_{2}\left(\mathrm{C}_{2} \mathrm{O}_{4}\right)_{2}$ ] (5) complex, showing the atom labeling for selected atoms and emphasizing the distorted octahedral coordination environments for the $\mathrm{Cd}^{2+}$ and $\mathrm{Ge}^{4+}$ metal centers. Non-hydrogen atoms composing the asymmetric unit are represented with thermal ellipsoids drawn at the $50 \%$ probability level. Hydrogen atoms were omitted for clarity. For selected bond lengths and angles for compounds 5 and $\mathbf{6}$ see Table 9. Symmetry transformation used to generate equivalent atoms: (i) $-x, y, 1.5-z$.

ambient temperature and ca. $268{ }^{\circ} \mathrm{C}$ a small continuous weight loss of about $0.8 \%$ confirms the presence of a small amount of water present in the structure (calculated 0.4 per formula unit), which agrees with the structural assumptions deduced during the single-crystal X-ray diffraction studies.

Neglecting the initial weight loss of compound $\mathbf{2 b}$ the thermal decomposition of all compounds can be roughly divided in two major stages: the first corresponds to release of a variable amount of phen ligands, and the second is attributed to decomposition of the $\left[\mathrm{Ge}\left(\mathrm{C}_{2} \mathrm{O}_{4}\right)_{3}\right]^{2-}$ anions. It is noteworthy to mention that the first thermal decomposition starts to settle in for compounds $\mathbf{1 a}$ and $\mathbf{1 b}$ at a lower temperature (around 205 and $201{ }^{\circ} \mathrm{C}$, respectively) than for the remaining materials (between 268 and $280{ }^{\circ} \mathrm{C}$ ) with this phenomenon being ascribed to the stronger oxidizing properties of $\mathrm{Cu}^{2+}$ when compared with the remaining transitionmetal centers. Nevertheless, in the $200-280{ }^{\circ} \mathrm{C}$ temperature range all materials release some phen ligands, usually between 2 and 3 molecules (1) ca. $2.1 ; \mathbf{1 b}$ ca. $2.5 ; \mathbf{3}$ ca. 2.3; 4 ca. 2.4). In the case of the $\mathrm{Fe}^{2+}$ compounds all phen molecules are thermally removed. Upon thermal decomposition of the $\left[\mathrm{Ge}\left(\mathrm{C}_{2} \mathrm{O}_{4}\right)_{3}\right]^{2-}$ anions, which usually starts to settle in around $300{ }^{\circ} \mathrm{C}$, the obtained residues are composed of a mixture of metallic oxides which, except for 1a (due to the presence of a small impurity in the as-synthesized material), are in good agreement with the expected stoichiometric quantities: for $\mathbf{1 b}, \mathrm{CuO}+\mathrm{GeO}_{2}$ (calculated $19.6 \%$, observed 20.1\%); for $\mathbf{2 a}$ and $\mathbf{2 b}, \mathrm{Fe}_{2} \mathrm{O}_{3}+\mathrm{GeO}_{2}$ (calculated $19.8 \%$ and $19.7 \%$, observed $20.0 \%$ and $20.6 \%$, respectively); for
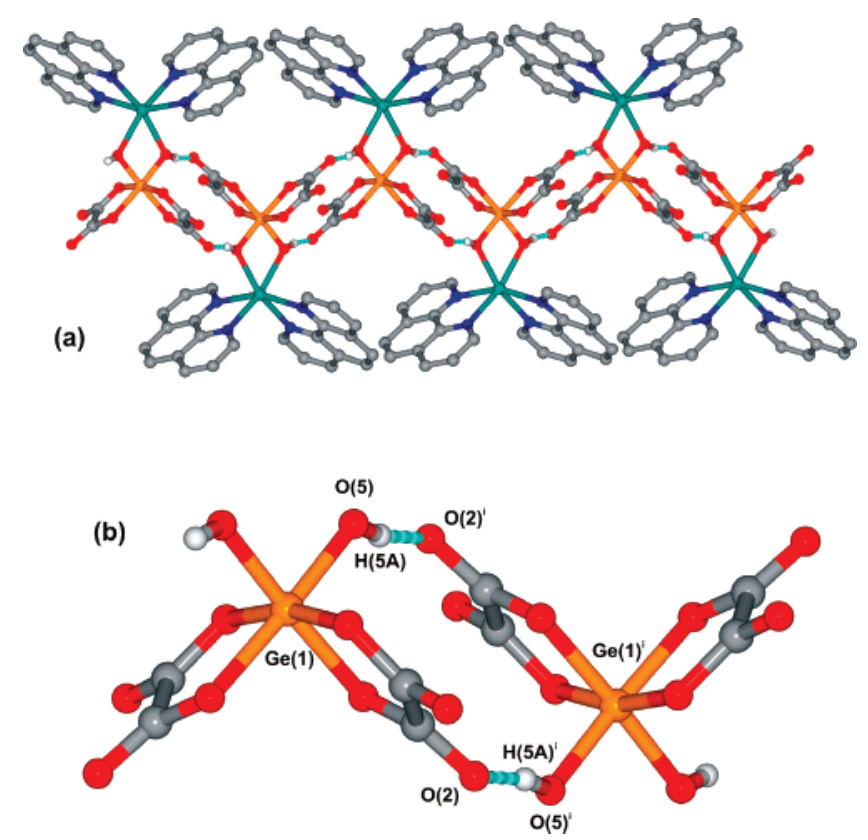

Figure 10. (a) Schematic representation of the parallel packing of individual $\left[\mathrm{MGe}(\mathrm{phen})_{2}\left(\mu_{2}-\mathrm{OH}\right)_{2}\left(\mathrm{C}_{2} \mathrm{O}_{4}\right)_{2}\right]$ complexes mediated by $\mathrm{O}-\mathrm{H} \cdots \mathrm{O}$ hydrogen bonds (dashed blue lines) and leading to formation of a one-dimensional supramolecular tape: $\infty^{2}\left[\mathrm{MGe}(\text { phen })_{2}\left(\mu_{2}-\mathrm{OH}\right)_{2}\left(\mathrm{C}_{2} \mathrm{O}_{4}\right)_{2}\right]$. (b) Magnification of a portion of the supramolecular tape emphasizing the $R^{2}{ }_{2}(12)$ graph set motif formed between two neighboring complexes and involving the $\mu_{2}$-bridging hydroxyl groups and coordinated oxalate anions. Geometrical details on $\mathrm{O}(5)-\mathrm{H}(5 \mathrm{~A}) \cdots \mathrm{O}(2)^{\mathrm{i}}$ : for $\mathbf{5}, d(\mathrm{O} \cdots \mathrm{O})=2.7699$ $(15) \AA$ with $\angle(\mathrm{OHO})=170.3(2)^{\circ}$; for $\mathbf{6}, d(\mathrm{O} \cdots \mathrm{O})=2.7791(13) \AA$ with $\angle(\mathrm{OHO})=168.0(1)^{\circ}$. Symmetry transformation used to generate equivalent atoms: (i) $-x,-y, 1-z$. For clarity, only hydrogen atoms involved in hydrogen-bonding interactions are represented.

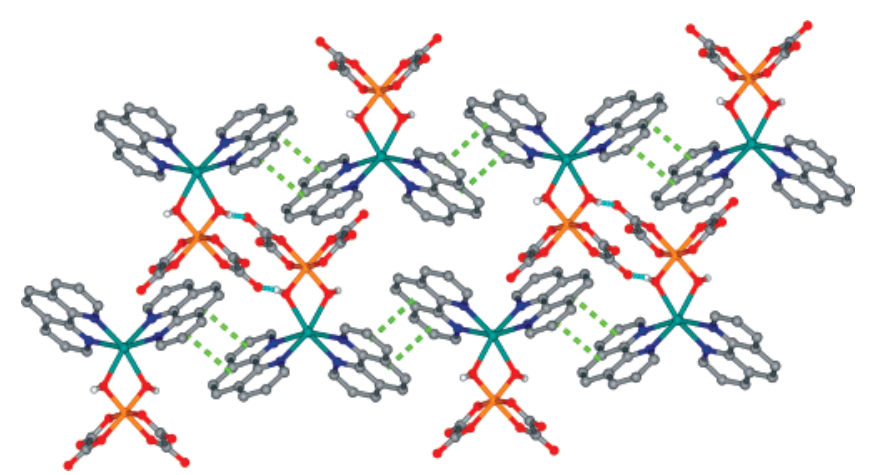

Figure 11. $\pi-\pi$ Interactions (dashed green lines) interconnecting neighboring ${ }_{\infty}^{2}\left[\mathrm{MGe}(\text { phen })_{2}\left(\mu_{2}-\mathrm{OH}\right)_{2}\left(\mathrm{C}_{2} \mathrm{O}_{4}\right)_{2}\right]$ supramolecular tapes. Hydrogenbonding interactions are represented as dashed blue lines, and hydrogen atoms belonging to the phen residues have been omitted for clarity.

3, $\mathrm{NiO}+\mathrm{GeO}_{2}$ (calculated $19.2 \%$, observed $19.6 \%$ ); for $\mathbf{4}$, $\mathrm{CoO}+\mathrm{GeO}_{2}$ (calculated $19.2 \%$, observed $20.0 \%$ ).

The thermal decomposition of compounds 5 and $\mathbf{6}$ starts with initial residual weight losses of about $0.6 \%$ and $0.5 \%$, respectively, which agrees well with the release of two water molecules per formula unit (calculated values of ca. $0.5 \%$ ). This can be attributed to destruction of the $\mu_{2}$ bridges which interconnecting the metals within the complex which, as clearly observed in the thermograms, occurs at a significantly higher temperature for $\mathbf{5}$ than for $\mathbf{6}$. Interestingly, the same is observed for the decomposition of the organic components of these compounds: while for 6 , at $600{ }^{\circ} \mathrm{C}$, all the material was entirely converted into the expected stoichiometric 
amount of $\mathrm{CuO}+\mathrm{GeO}_{2}$ (observed residual of $25.5 \%$, calculated of about $26.06 \%$ ), for $\mathbf{5}$ the decomposition is still occurring. The different kinetics associated with the thermal decomposition of $\mathbf{5}$ and $\mathbf{6}$ can be ascribed to the distinct physical-chemical properties of the $\mathrm{M}$ metals composing the bimetallic complexes with $\mathrm{Cu}^{2+}$ clearly promoting the decomposition.

Vibrational Spectroscopy. FT-IR and Raman spectra are particularly informative regarding the presence of the primary building blocks of the complexes, showing the characteristic bands of phen and oxalate organic ligands, hence fully supporting the chemical composition and structural details determined from the single-crystal X-ray diffraction studies. ${ }^{77}$ Specific band assignments for the most intense and diagnostic bands are collected in the Experimental Section, while the FT-IR spectra are provided as Supporting Information (Figure S3).

A number of bands located between 810 and $900 \mathrm{~cm}^{-1}$ are diagnostic of the presence of coordinated oxalate anions to $\mathrm{Ge}^{4+}$ and attributed to the $v(\mathrm{C}-\mathrm{C})$ and $v\left(\mathrm{Ge}-\mathrm{O}_{\text {oxalate }}\right)$ stretching vibrational modes. Several intense bands found in the ca. $1100-1520 \mathrm{~cm}^{-1}$ spectral region are characteristic of a number of vibrational modes of phen. A strong band centered at ca. $1735 \mathrm{~cm}^{-1}$, particularly evident in all spectra, strongly supports the existence of uncoordinated (and terminal) $\mathrm{C}=\mathrm{O}$ groups arising from the coordinated oxalate anions. Moreover, the $v_{\text {asym }}\left(-\mathrm{CO}_{2}^{-}\right)$and $v_{\text {sym }}\left(-\mathrm{CO}_{2}^{-}\right)$stretching vibrational bands of oxalate groups appear in the 16761580 and $1430-1310 \mathrm{~cm}^{-1}$ spectral regions, respectively, which correspond to $\Delta$ values between 246 and $270 \mathrm{~cm}^{-1}$, in good agreement with the observed anti,anti-chelate bidentate coordination fashion for these anionic moieties (see crystallographic description of the structures). ${ }^{78,79}$

In the case of $\mathbf{5}$ and $\mathbf{6}$, a broad band is markedly visible

(77) Socrates, G. Infrared Characteristic Group Frequencies-Tables and Charts, 2nd ed.; John Wiley \& Sons Ltd.: Baffins Lane, Chichester, 1994. above $3300 \mathrm{~cm}^{-1}$ (centered at ca. $3321 \mathrm{~cm}^{-1}$ for $\mathbf{5}$ and ca. $3396 \mathrm{~cm}^{-1}$ for 6$)$ and attributed to the characteristic $v(\mathrm{O}-$ $\mathrm{H})$ stretching vibrational band associated with the $\mu_{2}$-bridging hydroxyl groups involved in hydrogen bonds, in accord with the crystal structure. ${ }^{77}$

\section{Concluding Remarks}

The preparation, via typical hydrothermal approaches, of the first bimetallic complexes containing either $\left[\mathrm{Ge}\left(\mathrm{C}_{2} \mathrm{O}_{4}\right)_{3}\right]^{2-}$ or $\left[\mathrm{Ge}\left(\mathrm{C}_{2} \mathrm{O}_{4}\right)_{2}\right]$ with $\mathrm{M}$ cationic complexes of $1,10^{\prime}$-phenanthroline (phen) and their full structural description based on single-crystal X-ray diffraction investigations has been described. It was shown that the large chemical species composing each complex structure close pack in the solid state mediated by extensive subnetworks of intermolecular interactions which include, among others, strong $\mathrm{O}-\mathrm{H} \cdots \mathrm{O}$ and weak $\mathrm{C}-\mathrm{H} \cdots \mathrm{O}$ hydrogen-bonding interactions, $\mathrm{C}-\mathrm{H} \cdots \pi$ and $\pi-\pi$ contacts.

Acknowledgment. We are grateful to FEDER, POCI 2010, and Fundação para a Ciência e Tecnologia (FCT, Portugal) for their generous financial support, funding toward the purchase of the single-crystal diffractometer, and also postdoctoral research grants (no. SFRH/BPD/9309/2002 (to F.-N.S.) and SFRH/BPD/14410/2003 (to L.C.-S.)). We also thank the EPSRC (U.K.) and the University of Leeds for equipment funding.

Supporting Information Available: Crystallographic information as CIF files; Le Bail whole-powder-diffraction-pattern profile fitting of $\left[\mathrm{Co}(\text { phen })_{3}\right]\left[\mathrm{Ge}\left(\mathrm{C}_{2} \mathrm{O}_{4}\right)_{3}\right]$; thermograms and FT-IR spectra for all compounds. This material is available free of charge via the Internet at http://pubs.acs.org.

IC700507J

(78) Oldham, C. Carboxylates, Squarates and Related Species. In Comprehensive Coordination Chemistry, 1st ed.; Wilkinson, S. G., Ed.; Pergamon Press: New York, 1987; Vol. 2, pp 435-459.

(79) Deacon, G. B.; Phillips, R. J. Coord. Chem. Rev. 1980, 33, 227-250. 